\title{
Modified ion-Weibel instability as a possible source of wave activity at Comet 67P/Churyumov-Gerasimenko
}

\author{
Patrick Meier $^{1,2}$, Karl-Heinz Glassmeier ${ }^{1,3}$, and Uwe Motschmann ${ }^{2,4}$ \\ ${ }^{1}$ Institut für Geophysik und extraterrestrische Physik, TU Braunschweig, Mendelssohnstr. 3, 38106 Braunschweig, Germany \\ ${ }^{2}$ Institut für Theoretische Physik, TU Braunschweig, Mendelssohnstr. 3, 38106 Braunschweig, Germany \\ ${ }^{3}$ Max Planck Institute for Solar System Research, Justus-von-Liebig-Weg 3, 37077 Göttingen, Germany \\ ${ }^{4}$ German Aerospace Center (DLR), Institute of Planetary Research, Rutherfordstr. 2, 12489 Berlin, Germany \\ Correspondence to: Patrick Meier (patrick.meier@tu-bs.de)
}

Received: 11 May 2016 - Revised: 15 July 2016 - Accepted: 4 August 2016 - Published: 31 August 2016

\begin{abstract}
We analytically discuss wave excitation in a homogeneous three component plasma consisting of solar wind protons, electrons and a beam of cometary water ions applied to the plasma environment of comet 67P/ChuryumovGerasimenko. The resulting dispersion relations are studied in a solar wind rest frame, where a cometary current is solely generated by the water ion beam, and a cometary rest frame representing the rest frame of the Rosetta spacecraft. A modified ion-Weibel instability is excited by the cometary current and predominantly grows perpendicular to this current. The corresponding water ion mode is connected to a frequency of about $40 \mathrm{mHz}$ in agreement with wave measurements of Rosetta's magnetometer in the cometary rest frame. Furthermore, the superposition of the strongest growing waves result in a fan-like phase structure close to the comet.
\end{abstract}

Keywords. Magnetospheric physics (solar wind interaction with unmagnetized bodies) - space plasma physics (waves and instabilities)

\section{Introduction}

The study of waves in plasma environments is an extensive field in plasma physics. Special attention is paid to cometary magnetospheres, where plasma waves and turbulence are one of the most remarkable observations at comets like 1P/Halley, 21P/Giacobini-Zinner and 26P/GriggSkjellerup (Tsurutani and Smith, 1986; Yumoto et al., 1986; Neubauer et al., 1986; Glassmeier et al., 1989; Glassmeier and Neubauer, 1993; Volwerk et al., 2014). There, the interaction of the outgassing comets and the solar wind triggers ion ring-beam (Wu and Davidson, 1972) or non-gyrotropic phase space density instabilities (Motschmann and Glassmeier, 1993) resulting in the excitation of plasma waves.

Recently, investigations have been focused on comet 67P/Churyumov-Gerasimenko by the Rosetta mission (Glassmeier et al., 2007a). A new type of plasma wave with frequencies of tens of $\mathrm{mHz}$ has been detected by Rosetta's fluxgate magnetometer RPC-MAG (Glassmeier et al., 2007b) in the vicinity of this comet (Richter et al., 2015). At that time the comet was still beyond $2.7 \mathrm{AU}$ from the sun, the cometary activity just started and a strong magnetosphere was neither expected nor observed (Koenders et al., 2013; Rubin et al., 2014; Nilsson et al., 2015). In this environment the outgassing of the comet combined with the ionization of the water molecules generates a beam of cometary water ions possibly able to cause a type of beam instability. We discuss this scenario with a very simple ansatz of a cold, homogeneous three-component plasma, which is composed of magnetized solar wind protons, magnetized electrons and an unmagnetized beam of cometary water ions, in two different frames of reference. The first frame is the solar wind rest frame, where the cometary ion beam is the only moving plasma component. This frame simplifies the theoretical approach. The second frame is the cometary rest frame, which corresponds to the system, where the waves were measured by Rosetta, allowing comparisons with actual observations. Similar situations have already been studied (Chang et al., 1990; Sauer et al., 1998). However, these earlier studies do not discuss wave propagation perpendicular to the ambient magnetic field and the ion beam, which is the most important here. 
In this paper, we derive and discuss the analytic basics of this new type of low-frequency waves at the comet 67P/Churyumov-Gerasimenko. First, the applied model is explained and the dispersion relations are deduced in Sect. 2. These dispersion relations are mainly discussed in Sect. 3 regarding possible instabilities and phase structures. Finally, we present the conclusion in Sect. 4.

\section{Dispersion analysis applied to the plasma environment of 67P/Churyumov-Gerasimenko}

\subsection{Frames for the dispersion analysis}

For the dispersion analysis frames with resting comet and with resting solar wind are used. Finally, three frames are of advantage: the cometary rest frame CSEQ (comet-centred solar equatorial, Acton, 1996), the solar wind rest frame SW, and the tilted solar wind rest frame TSW.

CSEQ is the starting point as observations are presented just in this frame. As sketched in Fig. 1 (top) the origin is located in the comet, the $x$ axis points to the sun, the $y$ axis is in the solar equatorial plane and the $z$ axis completes the right-handed system. In this frame the solar wind velocity yields $\underline{\boldsymbol{u}}_{0 \mathrm{SW}}=-u_{0 \mathrm{SW}} \underline{\boldsymbol{e}}_{x}$. It points anti-sunward. For the ambient magnetic field $\underline{\boldsymbol{B}}_{0}$ we assume a pointing in $y$-direction as suggested by the Parker spiral, $\underline{\boldsymbol{B}}_{0}=B_{0} \underline{\boldsymbol{e}}_{y}$. Then, the motional electric field points along the $z$-direction, $\underline{\boldsymbol{E}}_{0}=E_{0} \underline{\boldsymbol{e}}_{z}$. Newborn cometary water ions launch their pick-up cycloidal motion along the motional electric field. As the gyroradius of cometary ions is large compared to the scale of the innermost coma these ions are essentially unmagnetized. They are controlled by the motional electric field providing us with $\underline{\boldsymbol{u}}_{0 \mathrm{c}}=u_{0 \mathrm{c}} \underline{\boldsymbol{e}}_{z}$ (Koenders et al., 2016). Otherwise the gyroradius of the newborn cometary electrons is very small. In the limit of massless electrons they are immediately picked up by the solar wind and thus their velocity is $\underline{\boldsymbol{u}}_{0 \mathrm{e}}=-u_{0 \mathrm{SW}} \underline{\boldsymbol{e}}_{x}$. We use this behaviour as an approach in our analysis, since it is still valid for electrons with finite mass on the length scales of our analysis. Thus, a single electron species composed of the solar wind and cometary electrons can be assumed. The resulting cometary electric current launched by the cometary plasma yields $\underline{\boldsymbol{j}}_{\mathrm{c}}=e n_{0 \mathrm{c}}\left(u_{0 \mathrm{c}} \underline{\boldsymbol{e}}_{z}-u_{0 \mathrm{SW}} \underline{\boldsymbol{e}}_{x}\right)$, where quasi-neutrality and singly charged ions are assumed. Additionally, in the $x z$ plane the azimuthal angle $\phi$ of the cur-

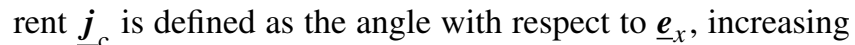
towards $\underline{\boldsymbol{e}}_{z}$. Thus, it is zero for a vector parallel to $\underline{\boldsymbol{e}}_{x}$.

A Galilean transformation along the $x$ axis with solar wind velocity catapults us into the solar wind rest frame SW as sketched in Fig. 1 (mid). Now, the electrons and solar wind protons are at rest and the comet moves with the velocity $\underline{\boldsymbol{u}}_{\mathrm{comet}}=-\underline{\boldsymbol{u}}_{0 \mathrm{SW}}$. The cometary ion velocity keeps its $z-$ component, but it takes on an additional $x$-component, $\underline{\boldsymbol{u}}_{\mathrm{c}}=$ $-u_{0 \mathrm{SW}} \underline{\boldsymbol{e}}_{x}+u_{0 \mathrm{c}} \underline{\boldsymbol{e}}_{z}$. In this frame the cometary current is only carried by the cometary ions, $\underline{\boldsymbol{j}}_{\mathrm{c}}=e n_{0 \mathrm{c}}\left(-u_{0 \mathrm{SW}} \underline{\boldsymbol{e}}_{x}+u_{0 \mathrm{c}} \underline{\boldsymbol{e}}_{z}\right)$.
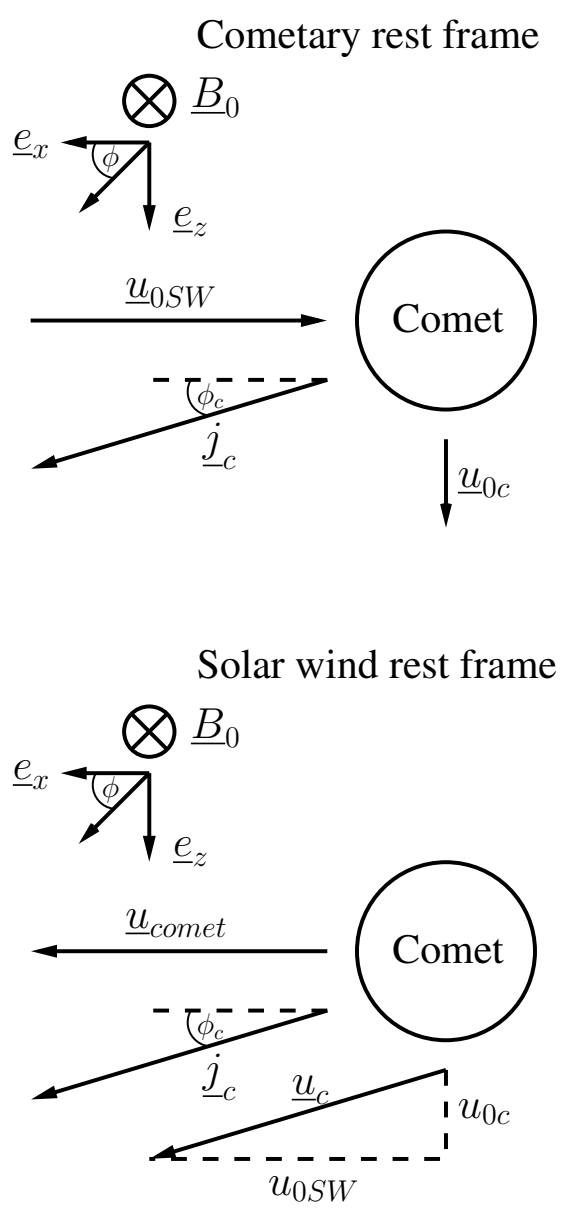

Tilted solar wind rest frame

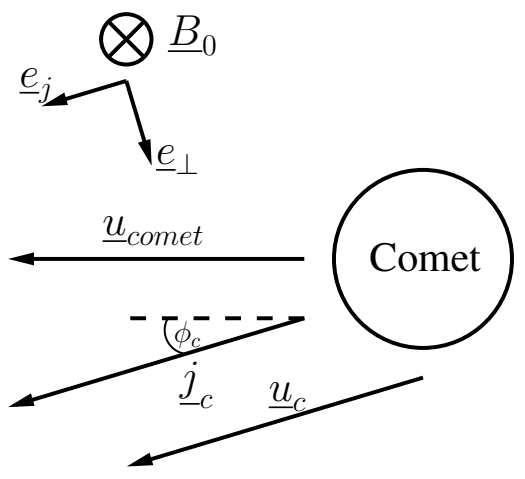

Figure 1. Illustration of the cometary rest frame CSEQ (top), solar wind rest frame SW (mid) and tilted solar wind rest frame TSW (bottom). Characteristic velocities of the solar wind $\underline{\boldsymbol{u}}_{0 \mathrm{SW}}=$ $-u_{0 \mathrm{SW}} \underline{\boldsymbol{e}}_{x}$, cometary ions $\underline{\boldsymbol{u}}_{c 0}=u_{0 \mathrm{c}} \underline{\boldsymbol{e}}_{z}$ and $\underline{\boldsymbol{u}}_{\mathrm{c}}=-u_{0 \mathrm{SW}} \underline{\boldsymbol{e}}_{x}+u_{0 \mathrm{c}} \underline{\boldsymbol{e}}_{z}$ and the comet $\underline{\boldsymbol{u}}_{\mathrm{comet}}=-\underline{\boldsymbol{u}}_{0 \mathrm{SW}}$ are shown in the different frames. Additionally, the ambient magnetic field $\underline{\boldsymbol{B}}_{0}=B_{0} \underline{\boldsymbol{e}}_{y}$, the cometary current $\underline{\boldsymbol{j}}_{\mathrm{c}}=n_{0 \mathrm{c}} \underline{\boldsymbol{u}}_{\mathrm{c}}$, the definition of the azimuthal angle $\phi$ and the characteristic angle $\phi_{\mathrm{c}}=\arctan \left(u_{0 \mathrm{c}} / u_{0 \mathrm{SW}}\right)$ between SW and TSW are sketched. 
Table 1. List of plasma characteristics in the environment of P67/Churyumov-Gerasimenko at heliocentric distances of about $3 \mathrm{AU}$ (Hansen et al., 2007; Richter et al., 2015): the velocities are with respect to CSEQ.

\begin{tabular}{llr}
\hline Parameter & Symbol & Value \\
\hline Magnetic field & $B_{0}$ & $2.5 \mathrm{nT}$ \\
Electron mass & $m_{\mathrm{e}}$ & $9.1 \times 10^{-31} \mathrm{~kg}$ \\
Proton mass & $m_{\mathrm{p}}$ & $1.7 \times 10^{-27} \mathrm{~kg}$ \\
Water ion mass & $m_{\mathrm{c}}$ & $18 m_{\mathrm{p}}$ \\
Electron number density & $n_{0 \mathrm{e}}$ & $2.0 \mathrm{~cm}^{-3}$ \\
Proton number density & $n_{0 \mathrm{p}}$ & $1.0 \mathrm{~cm}^{-3}$ \\
Water ion number density & $n_{0 \mathrm{c}}$ & $1.0 \mathrm{~cm}^{-3}$ \\
Solar wind velocity & $u_{0 \mathrm{SW}}$ & $4.0 \times 10^{2} \mathrm{~km} \mathrm{~s}^{-1}$ \\
Water ion velocity & $u_{0 \mathrm{c}}$ & $4.0 \times 10^{1} \mathrm{~km} \mathrm{~s}^{-1}$ \\
Electron plasma frequency & $\omega_{\mathrm{e}}$ & $8.0 \times 10^{4} \mathrm{~s}^{-1}$ \\
Proton plasma frequency & $\omega_{\mathrm{p}}$ & $1.3 \times 10^{3} \mathrm{~s}^{-1}$ \\
Water ion plasma frequency & $\omega_{\mathrm{c}}$ & $3.1 \times 10^{2} \mathrm{~s}^{-1}$ \\
Electron gyro-frequency & $\Omega_{\mathrm{e}}$ & $-4.4 \times 10^{2} \mathrm{~s}^{-1}$ \\
Proton gyro-frequency & $\Omega_{\mathrm{p}}$ & $2.4 \times 10^{-1} \mathrm{~s}^{-1}$ \\
Water ion gyro-frequency & $\Omega_{\mathrm{c}}$ & $1.3 \times 10^{-2} \mathrm{~s}^{-1}$ \\
\hline
\end{tabular}

Note that this current is the same current as in CSEQ, i.e. it is independent of the rest frame.

For the analytical treatment of the dispersion relation the introduction of a tilted solar wind rest frame TSW, with the new $x$ axis pointing along the current $\boldsymbol{j}_{c}$, is most convenient. TWS is sketched in Fig. 1 (bottom). The basis vectors in this frame are called $\left\{\underline{\boldsymbol{e}}_{j}, \underline{\boldsymbol{e}}_{B}, \underline{\boldsymbol{e}}_{\perp}\right\}$ with unchanged $y$ axis. The tilt is realized by a rotation around the $y$ axis over an angle

$\phi_{\mathrm{c}}=\arctan \left(\frac{u_{0 \mathrm{c}}}{u_{0 \mathrm{SW}}}\right)$

providing us

$\underline{\boldsymbol{e}}_{j}=\cos \phi_{\mathrm{c}} \underline{\boldsymbol{e}}_{x}+\sin \phi_{\mathrm{c}} \underline{\boldsymbol{e}}_{z}$

$\underline{\boldsymbol{e}}_{B}=\underline{\boldsymbol{e}}_{y}$

$\underline{\boldsymbol{e}}_{\perp}=-\sin \phi_{\mathrm{c}} \underline{\boldsymbol{e}}_{x}+\cos \phi_{\mathrm{c}} \underline{\boldsymbol{e}}_{z}$.

The $\underline{\boldsymbol{e}}_{\perp}$ axis is defined as perpendicular to the cometary current $\underline{\boldsymbol{j}}_{\mathrm{c}}$ and the ambient magnetic field $\underline{\boldsymbol{B}}_{0}$ completing the right-handed system.

The equations of the dispersion relation are related just to the TWS. Later, the results are transformed back to the CSEQ for the discussion and interpretation.

\subsection{Dispersion analysis in the tilted solar wind rest frame}

Now, the general dispersion tensor $\underline{\underline{D}}$ is derived by linearizing Maxwell's equations as can be found in e.g. Baumjohann and Treumann (1997) and is also discussed in detail in Appendix A. The components of the dispersion tensor are speci- fied for the cold, three-component plasma consisting of magnetized electrons, solar wind protons and a beam of unmagnetized cometary water ions in TSW by

$$
\begin{aligned}
& D_{j j}=1-\frac{c^{2}\left(k_{B}^{2}+k_{\perp}^{2}\right)}{\omega^{2}}-\frac{\omega_{e}^{2}}{\omega^{2}-\Omega_{e}^{2}} \\
& -\frac{\omega_{p}^{2}}{\omega^{2}-\Omega_{p}^{2}}-\frac{\omega_{\mathrm{c}}^{2}}{\omega^{2}} \frac{\omega^{2}+\left(k_{B}^{2}+k_{\perp}^{2}\right) u_{\mathrm{c}}^{2}}{\left(\omega-k_{j} u_{\mathrm{c}}\right)^{2}} \\
& D_{B B}=1-\frac{c^{2}\left(k_{j}^{2}+k_{\perp}^{2}\right)}{\omega^{2}}-\frac{\omega_{e}^{2}+\omega_{p}^{2}+\omega_{\mathrm{c}}^{2}}{\omega^{2}} \\
& D_{\perp \perp}=1-\frac{c^{2}\left(k_{j}^{2}+k_{B}^{2}\right)}{\omega^{2}}-\frac{\omega_{e}^{2}}{\omega^{2}-\Omega_{e}^{2}}-\frac{\omega_{p}^{2}}{\omega^{2}-\Omega_{p}^{2}}-\frac{\omega_{\mathrm{c}}^{2}}{\omega^{2}} \\
& D_{j B}=\frac{c^{2} k_{j} k_{B}}{\omega^{2}}-\frac{\omega_{\mathrm{c}}^{2}}{\omega^{2}} \frac{k_{B} u_{\mathrm{c}}}{\omega-k_{j} u_{\mathrm{c}}}=D_{B j} \\
& D_{j \perp}=\frac{c^{2} k_{j} k_{\perp}}{\omega^{2}}-\frac{\omega_{\mathrm{c}}^{2}}{\omega^{2}} \frac{k_{\perp} u_{\mathrm{c}}}{\omega-k_{j} u_{\mathrm{c}}} \\
& -\frac{i}{\omega}\left(\frac{\Omega_{e} \omega_{e}^{2}}{\omega^{2}-\Omega_{e}^{2}}+\frac{\Omega_{p} \omega_{p}^{2}}{\omega^{2}-\Omega_{p}^{2}}\right) \\
& D_{\perp j}=\frac{c^{2} k_{j} k_{\perp}}{\omega^{2}}-\frac{\omega_{\mathrm{c}}^{2}}{\omega^{2}} \frac{k_{\perp} u_{\mathrm{c}}}{\omega-k_{j} u_{\mathrm{c}}} \\
& +\frac{i}{\omega}\left(\frac{\Omega_{e} \omega_{e}^{2}}{\omega^{2}-\Omega_{e}^{2}}+\frac{\Omega_{p} \omega_{p}^{2}}{\omega^{2}-\Omega_{p}^{2}}\right) \\
& D_{B \perp}=\frac{c^{2} k_{B} k_{\perp}}{\omega^{2}}=D_{\perp B}
\end{aligned}
$$

with plasma frequencies $\omega_{\alpha}=\sqrt{q_{\alpha}^{2} n_{0 \alpha} /\left(\varepsilon_{0} m_{\alpha}\right)}$ and gyrofrequencies $\Omega_{\alpha}=q_{\alpha}\left|\underline{\boldsymbol{B}}_{0}\right| / m_{\alpha}$ of the respective plasma species $\alpha \in\{e, p, c\}$ (see Table 1). Similar expressions were derived by Chang et al. (1990) for a two-species plasma with magnetized electrons and an unmagnetized ion beam. In a study by Sauer et al. (1998) a second, heavy ion species was considered as a beam, but the deduced dielectric tensor elements differ from our results and from what is expected from Chang et al. (1990). Furthermore, wave propagation perpendicular to the magnetic field and the beam was neglected by both studies. The dispersion relation results from

$\operatorname{det} \underline{\underline{D}}(\omega, \underline{\boldsymbol{k}})=0$

that is numerically studied in the next section. Additionally, the dispersion relation is further analytically discussed in Appendix B yielding cut-off and resonance frequencies, which approach the known frequencies of a two component electron-proton plasma in the limit of vanishing cometary ions. In particular, the electron and proton gyrofrequencies are found as resonance frequencies of modified R- and L-modes $\left(\omega_{R / L \text {, res }}= \pm \Omega_{e / p}\right)$, respectively. Furthermore, a new, purely imaginary resonance frequency $\omega_{c}$, res $=$ $\pm i \omega_{\mathrm{c}} u_{\mathrm{c}} / c$ results from the water ion beam for wave propa- 


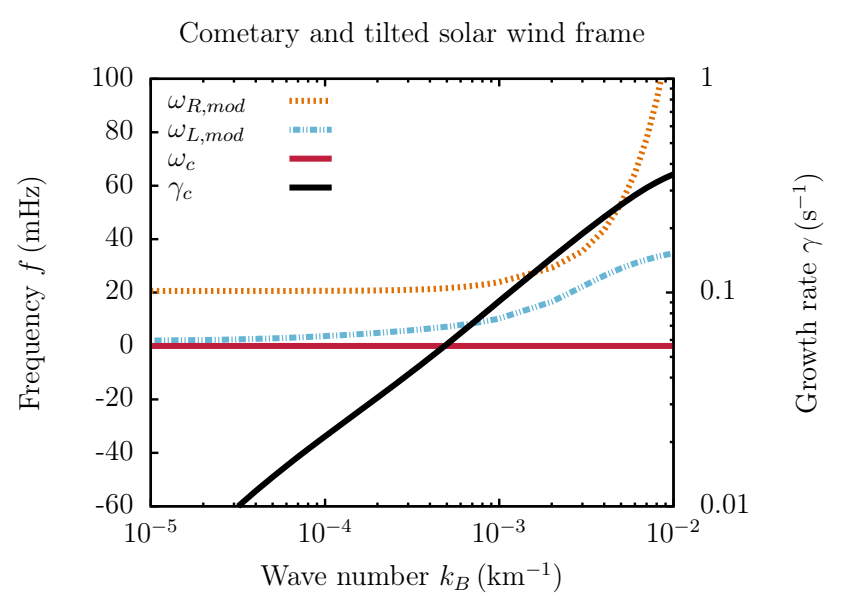

Figure 2. Wave propagation parallel to the ambient magnetic field $\underline{\boldsymbol{B}}_{0}$. The frequencies and growth rates of the dispersion relation are shown. Since the Doppler shift does not affect this propagation, the same dispersion results from TSW and the cometary rest frame. The new mode regarding the water ions yields only a growth rate $\gamma_{\mathrm{c}}$ (black) with an upper limit of $\gamma_{c}$, res $\approx 0.42 \mathrm{~s}^{-1}$ but no frequency $\omega_{\mathrm{c}}$ (red), while the modified R- (orange) and L-modes (light blue) are $\omega_{R}$, mod and $\omega_{L}$, mod, respectively. The negative branches are not shown, since they are mirror symmetric to the $k_{B}$ axis.

gation parallel to the ambient magnetic field. For wave propagation perpendicular to the ambient magnetic field a modified O-mode is identified as well as a modified lower-hybrid frequency as resonance frequency of a X-mode. Although no further resonance frequency is found for wave propagation parallel to the cometary current, there is also a purely imaginary resonance frequency due to the water ions for wave propagation perpendicular to the current and the magnetic field.

\section{Discussion}

\subsection{Instabilities}

In the previous section and Appendix B the dispersion relations were deduced, which are in agreement with the known wave modes in the limit of vanishing cometary ions. Now, we discuss the dispersion relations in particular regarding instabilities for three characteristic propagation directions: parallel to the ambient magnetic field $\underline{\boldsymbol{B}}_{0}$ (Fig. 2), parallel to the cometary current $\underline{j}_{c}$ (Fig. 4) and perpendicular to both of them (Fig. 3). Since the dispersion relations can be complex for $\omega=\omega_{\Re}+i \gamma$, the frequency $f=\omega_{\Re} / 2 \pi$ and growth rate $\gamma$ are separated to the left and right scales of the figures, respectively. The modified L-, R- and X-modes are also shown for completeness.

We start our discussion with wave propagation parallel to the ambient magnetic field $\underline{\boldsymbol{B}}_{0}$ (Fig. 2) based on the disper- sion relation Eq. (B9)

$$
\begin{aligned}
& \frac{c^{2} k_{B}^{2}}{\omega^{2}}=-\frac{\omega_{e}^{2}}{\omega^{2}-\Omega_{e}^{2}}-\frac{\omega_{p}^{2}}{\omega^{2}-\Omega_{p}^{2}}-\frac{\omega_{\mathrm{c}}^{2}}{\omega^{4}}\left(\omega^{2}+k_{B}^{2} u_{\mathrm{c}}^{2}\right) \\
& -\frac{\frac{2 \omega^{2}}{\omega_{\mathrm{c}}^{2} k_{B}^{2} u_{\mathrm{c}}^{2}}\left(\frac{\Omega_{e} \omega_{e}^{2}}{\omega^{2}-\Omega_{e}^{2}}+\frac{\Omega_{p} \omega_{p}^{2}}{\omega^{2}-\Omega_{p}^{2}}\right)^{2}}{1 \pm \sqrt{1+4 \frac{\omega^{6}}{\omega_{\mathrm{c}}^{4} k_{B}^{4} u_{\mathrm{c}}^{4}}\left(\frac{\Omega_{e} \omega_{e}^{2}}{\omega^{2}-\Omega_{e}^{2}}+\frac{\Omega_{p} \omega_{p}^{2}}{\omega^{2}-\Omega_{p}^{2}}\right)^{2}}} .
\end{aligned}
$$

Since the Doppler shift does not affect these waves, the dispersion relations are the same for TSW and the cometary rest frame. Furthermore, the modes are mirror symmetric to the $k_{B}$ axis, so only the positive branches are shown. Besides the modified L- (light blue) and R-modes (orange), we deduced an imaginary resonance for the cometary ion mode from Eq. (B11), i.e. there is only a growth rate (black) with an upper limit $\gamma_{c \text {, res }} \approx 0.42 \mathrm{~s}^{-1} \approx 31.37 \Omega_{\mathrm{c}}$, but the frequency is zero (red). For a decreasing wave number the growth rate also declines, but a finite frequency does not appear. Hence, perturbations of the plasma can grow, but do not propagate parallel to the ambient magnetic field. A similar result was found by Chang et al. (1990) for a two component electron-ion plasma with an ion beam, where this purely growing mode was associated with an ion-Weibel instability. A Weibel instability is a self-excited electromagnetic wave in a nearly homogeneous plasma generated by a electron velocity distribution, which is sufficiently anisotropic (Weibel, 1959; Fried, 1959; Lui et al., 1991). This wave can even be excited in a cold plasma without an ambient magnetic field yielding a purely imaginary dispersion perpendicular to the predominant velocity direction. Weibel instabilities are also excited in relativistic plasmas (Yoon and Davidson, 1987) and for relativistic shocks (Milosavljević et al., 2006). This type of instability is even derived from electroweak (Silva et al., 2000) and quark-gluonen plasmas (Arnold and Moore, 2006; Strickland, 2007) and is considered as an alternative source for quasi-stationary magnetic fields besides dynamos (Treumann and Baumjohann, 2012). Furthermore, in gyrotropic or unmagnetized plasmas the existence of a pure growing mode has also been shown based on general linear fluctuation theory and fluctuation-dissipation theorems (Schlickeiser and Yoon, 2015; Schlickeiser et al., 2015). In our analysis of a classical plasma a velocity anisotropy is carried by the cometary water ion beam in TSW and the instability is modified by the additional plasma component of magnetized protons resulting in a modified ion-Weibel instability.

Next, we discuss the wave propagation perpendicular to both the ambient magnetic field $\underline{\boldsymbol{B}}_{0}$ and cometary current $\underline{\boldsymbol{j}}_{\mathrm{c}}$ $\left(\phi \approx 96^{\circ}\right.$, see Fig. 1 for definition of the azimuth $\left.\phi\right)$, which has been typically neglected by similar studies (Wu et al., 

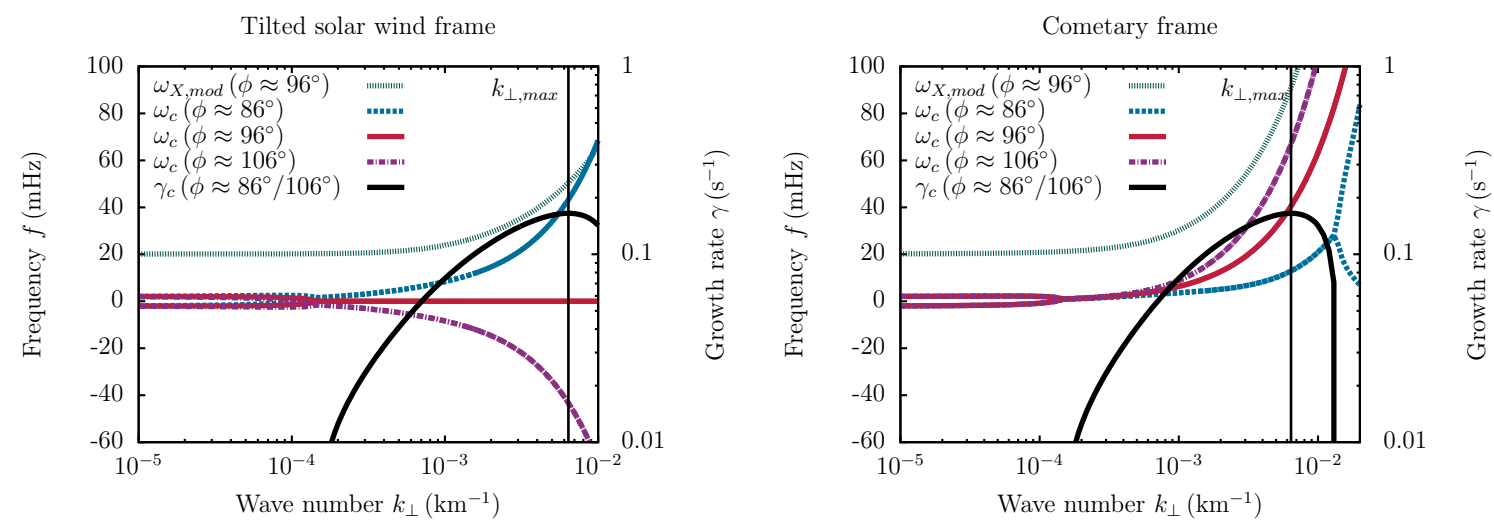

Figure 3. Wave propagation perpendicular to the cometary current $\underline{\boldsymbol{j}}_{\mathrm{c}}\left(\phi \approx 96^{\circ}\right)$ and the ambient magnetic field $\underline{\boldsymbol{B}}_{0}$. The modified X-mode (green) is labelled $\omega_{X}$, mod and both branches of the cometary ion mode $\omega_{\mathrm{c}}$ (red) are shown. Additionally, the cometary ion branches close to the perpendicular direction $\left(\phi \approx 86^{\circ} / 106^{\circ}\right)$ (blue/violet) and their growth rate (black) with a maximum at $k_{\perp, \text { max }} \approx 6.4 \times 10^{-3} \mathrm{~km}^{-1}$ $\left(\lambda_{\max } \approx 980 \mathrm{~km}\right)$ are presented, since for $\phi \approx 96^{\circ}$ the growth rate (not shown) only approaches an upper limit $\gamma_{c}$, res $\approx 0.40 \mathrm{~s}^{-1}$. TSW and the cometary rest frame are shown in the left and right panels, respectively.
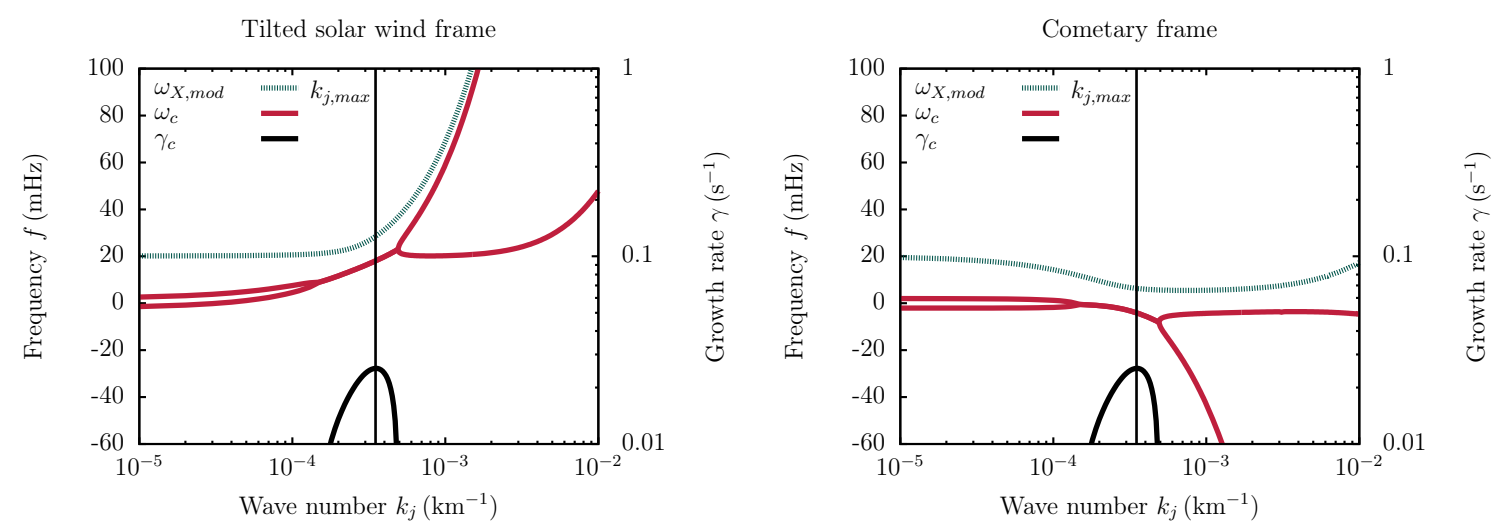

Figure 4. Wave propagation parallel to the cometary current $\underline{\boldsymbol{j}}_{\mathrm{c}}$ analogous to Fig. 3. A maximum of the growth rate $\gamma_{\mathrm{c}}$ (black) is found at wave number $k_{j, \max } \approx 3.5 \times 10^{-4} \mathrm{~km}^{-1}\left(\lambda_{\max } \approx 18000 \mathrm{~km}\right)$ with finite frequencies in the water ion branches (red). While the frequency is positive in TSW (left), the wave propagates in opposite direction due to the Doppler shift in the cometary rest frame (right) resulting in a negative frequency.

1983; Chang et al., 1990; Sauer et al., 1998), resulting from

$$
\begin{aligned}
& \frac{c^{2} k_{\perp}^{2}}{\omega^{2}}= \\
& -\frac{\left(\frac{\omega_{e}^{2}}{\omega^{2}-\Omega_{e}^{2}}+\frac{\omega_{p}^{2}}{\omega^{2}-\Omega_{p}^{2}}+\frac{\omega_{\mathrm{c}}^{2}}{\omega^{2}}\right)^{2}-\frac{1}{\omega^{2}}\left(\frac{\Omega_{e} \omega_{e}^{2}}{\omega^{2}-\Omega_{e}^{2}}+\frac{\Omega_{p} \omega_{p}^{2}}{\omega^{2}-\Omega_{p}^{2}}\right)^{2}}{\left(1+\frac{\omega_{\mathrm{c}}^{2}}{\omega^{2}} \frac{u_{\mathrm{c}}^{2}}{c^{2}}\right)\left(\frac{\omega_{e}^{2}}{\omega^{2}-\Omega_{e}^{2}}+\frac{\omega_{p}^{2}}{\omega^{2}-\Omega_{p}^{2}}+\frac{\omega_{\mathrm{c}}^{2}}{\omega^{2}}\right)-\frac{\omega_{\mathrm{c}}^{4}}{\omega^{4}} \frac{u_{\mathrm{c}}^{2}}{c^{2}}} .
\end{aligned}
$$

Besides the modified X-mode (green, Fig. 3), the positive and negative branches of the cometary ion mode (red) are shown. The propagation is affected by the Doppler shift, so the modes in TSW and the cometary rest frame are separated in the left and right panels of Fig. 3, respectively. As already seen for propagation parallel to $\underline{\boldsymbol{B}}_{0}$ the cometary ion mode also yields a pure growth rate in the resonance with $\gamma_{c \text {, res }} \approx 0.40 \mathrm{~s}^{-1} \approx 29.88 \Omega_{\mathrm{c}}$ in $\mathrm{TSW}$, so we also include propagation close to that direction $\left(\phi \approx 86^{\circ}\right.$, blue and $\phi \approx 106^{\circ}$, violet). The modes of these directions are symmetric in TSW and result in a maximum for the growth rate (black) at $k_{\perp, \max } \approx 6.4 \times 10^{-3} \mathrm{~km}^{-1}$, corresponding to a wave length $\lambda_{\max } \approx 980 \mathrm{~km}$. The existence of a maximum of the growth rate is connected to the splitting of the branches of the cometary ion mode for $k_{\perp} \gtrsim 1.4 \times 10^{-2} \mathrm{~km}^{-1}$. In contrast, the splitting is not found for $\phi \approx 96^{\circ}$, but an upper limit of the growth rate is approached for large wave numbers. Furthermore, the positive and negative branches for $\phi \approx 86^{\circ} / 96^{\circ} / 106^{\circ}$ start stably at their respective cut-off frequencies and become unstable at $k_{\perp} \gtrsim 1.5 \times 10^{-4} \mathrm{~km}^{-1}$. Since the branch for propagation perpendicular to $\underline{\boldsymbol{B}}_{0}$ and $\boldsymbol{j}$ is wrapped by the two branches close to this, we use $k_{\perp, \max } \approx$ $6.4 \times 10^{-3} \mathrm{~km}^{-1}$ as lower limit for the former. According to 

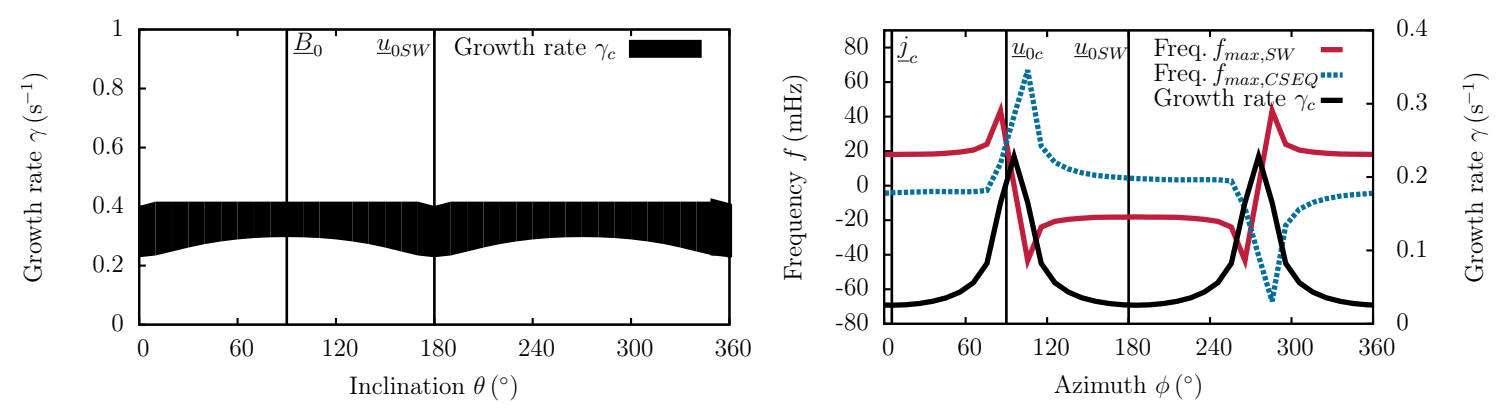

Figure 5. Left: estimated growth rate $\gamma_{c}$ range in the plane perpendicular to the cometary current $\underline{j}_{c}$. The black bar corresponds to the range between the estimated lower limit of $k_{\max }=6.4 \times 10^{-3} \mathrm{~km}^{-1}$ and the asymptotic upper limit of the growth rate. Since in this plane no real frequency is given in SW, only the growth rate is shown. Right: comparison between the frequencies with maximum growth rate of $\omega_{\mathrm{c}}$ in SW (red) and CSEQ (blue) with variation of the azimuth $\phi$ in the plane perpendicular to the ambient magnetic field $\underline{\boldsymbol{B}}_{0}$. In addition, the corresponding maxima of the growth rate (black) are shown. For propagation $\underline{k} \perp \underline{j}_{\mathrm{c}}$ the lower limit of $k_{\max }=6.4 \times 10^{-3} \mathrm{~km}^{-1}$ is assumed due to the missing maximum. Additionally, characteristic propagation directions are marked in both panels.

this estimation, the lower limit of the growth rate is $\gamma_{c, \max } \approx$ $0.24 \mathrm{~s}^{-1} \approx 17.93 \Omega_{\mathrm{c}}$. The corresponding frequency is zero $\left(\phi \approx 96^{\circ}\right)$ in TSW, while close to this $\left(\phi \approx 86^{\circ} / 106^{\circ}\right)$ the frequency is $f \approx \pm 43 \mathrm{mHz}$. The sign follows from the wave vector component parallel to the cometary current. In the cometary rest frame the frequency range is Doppler shifted to $13 \mathrm{mHz} \lesssim f \lesssim 67 \mathrm{mHz}$ with $f_{\max , \mathrm{CSEQ}} \approx 41 \mathrm{mHz}$ for $\phi \approx$ $96^{\circ}$. These frequencies well agree with the "singing" of comet 67P/Churyumov-Gerasimenko, which was measured by Rosetta's magnetometer (Richter et al., 2015). Hence, the modified ion-Weibel instability, which is actually excited by the cometary current $\underline{j}_{\mathrm{c}}$, is the most probable source for the comet's song.

Last, wave propagation parallel to the cometary current $\underline{j}_{\mathrm{c}}\left(\phi \approx 6^{\circ}\right)$ is discussed based on the dispersion relation $\overline{\mathrm{Eq}} \mathrm{c}$ (B17)

$$
\begin{aligned}
& \frac{c^{2} k_{j}^{2}}{\omega^{2}}= \\
& -\frac{\left(\frac{\omega_{e}^{2}}{\omega^{2}-\Omega_{e}^{2}}+\frac{\omega_{p}^{2}}{\omega^{2}-\Omega_{p}^{2}}+\frac{\omega_{c}^{2}}{\omega^{2}}\right)\left(\frac{\omega_{c}^{2}}{\omega^{2}-\Omega_{e}^{2}}+\frac{\omega_{p}^{2}}{\omega^{2}-\Omega_{p}^{2}}+\frac{\omega_{c}^{2}}{\left(\omega-\mu_{j} u_{c}\right)^{2}}\right)-\frac{1}{\omega^{2}}\left(\frac{\Omega_{e} \omega_{e}^{2}}{\omega^{2}-\Omega_{e}^{2}}+\frac{\Omega_{p} \omega_{p}^{2}}{\omega^{2}-\Omega_{p}^{2}}\right)^{2}}{\frac{\omega_{e}^{2}}{\omega^{2}-\Omega_{e}^{2}}+\frac{\omega_{p}^{2}}{\omega^{2}-\Omega_{p}^{2}}+\frac{\omega_{c}^{2}}{\left(\omega-k_{j} u_{c}\right)^{2}}} .
\end{aligned}
$$

Since this propagation is also affected by the Doppler shift, TSW and the cometary rest frame are again displayed in the left and right panels of Fig. 4, respectively. A growth rate occurs in a finite interval $1.5 \times$ $10^{-4} \mathrm{~km}^{-1} \lesssim k_{j} \lesssim 4.9 \times 10^{-4} \mathrm{~km}^{-1}$ and has a maximum at $k_{j, \max } \approx 3.5 \times 10^{-4} \mathrm{~km}^{-1}$, corresponding to a wave length $\lambda_{\max } \approx 18000 \mathrm{~km}$, with $\gamma_{c, \text { max }} \approx 0.025 \mathrm{~s}^{-1} \approx 1.87 \Omega_{\mathrm{c}}$. The positive and negative branches again start from their corresponding cut-off frequencies, rise with increasing wave number and couple at $k_{j} \approx 1.5 \times 10^{-4} \mathrm{~km}^{-1}$ until they split at $k_{j} \approx 4.9 \times 10^{-4} \mathrm{~km}^{-1}$ in TSW. In the cometary rest frame the branches turn to negative values, couple and also decouple for negative frequencies. The different signs again mean different propagation directions. The growing waves propagate along the cometary current in TSW. In contrast, the waves go along with the solar wind and propagate in opposite direction of the current in the cometary rest frame. The frequencies corresponding to $k_{j, \max }$ are $f_{\text {max }}$, TSW $\approx 17 \mathrm{mHz}$ and $f_{\text {max }, \mathrm{CSEQ}} \approx-4.1 \mathrm{mHz}$ in the respective frames. Despite the differences in the dielectric tensor (see Sect. 2), a qualitatively similar trend in the dispersion relation was mentioned for oblique propagation in the plane spanned by $\underline{\boldsymbol{B}}_{0}$ and $\boldsymbol{j}_{\mathrm{c}}$, but not further discussed by these authors (Sauer et al., 1998).

So far we derived growth rates parallel and perpendicular to the cometary current, but not parallel to the ambient magnetic field. Therefore, with the lower limit for the wave number $k_{\perp, \max } \approx 6.4 \times 10^{-3} \mathrm{~km}^{-1}$ from perpendicular propagation we go back to the wave vector parallel to the ambient magnetic field and estimate a lower limit for the growth rate of about $\gamma_{c, \max } \approx 0.30 \mathrm{~s}^{-1} \approx 22.41 \Omega_{\mathrm{c}}$. The discussion with respect to the three axes indicated that the growth rate is roughly constant in the plane perpendicular to the cometary current, but about 1 order of magnitude larger than for a wave vector parallel to the current. This is confirmed by Fig. 5. In the left panel the estimated growth rate range (black) is shown in the plane perpendicular to the current as a function of the inclination $\theta$. In the $\perp B$ plane the inclination angle $\theta$ of the wave vector $\underline{k}$ is defined as the angle with respect to $\underline{\boldsymbol{e}}_{\perp}$, increasing towards $\underline{\boldsymbol{e}}_{B}$. Since in this plane the dispersion relation does not yield a real frequency in SW, only the growth rate is shown that is roughly constant as expected. Furthermore, this growth rate is clearly beyond a possible water ion gyro-frequency $\Omega_{\mathrm{c}} \approx 1.3 \times 10^{-2} \mathrm{~s}^{-1}$ (Table 1) in compliance with the assumption of an unmagnetized cometary ion beam. This means that the modified ion-Weibel instability is already well established before gyration effects arise, in contrast to ion cyclotron (Drummond and Rosenbluth, 1962; Kindel and Kennel, 1971), fire-hose (Gary et al., 1976) or mirror instabilities (Gary, 1992) with theoretical growth rates $\sim 10^{-2} \Omega_{\mathrm{c}}$. However, in the terrestrial magnetosheath mirror modes with growth rates 1 order of magni- 

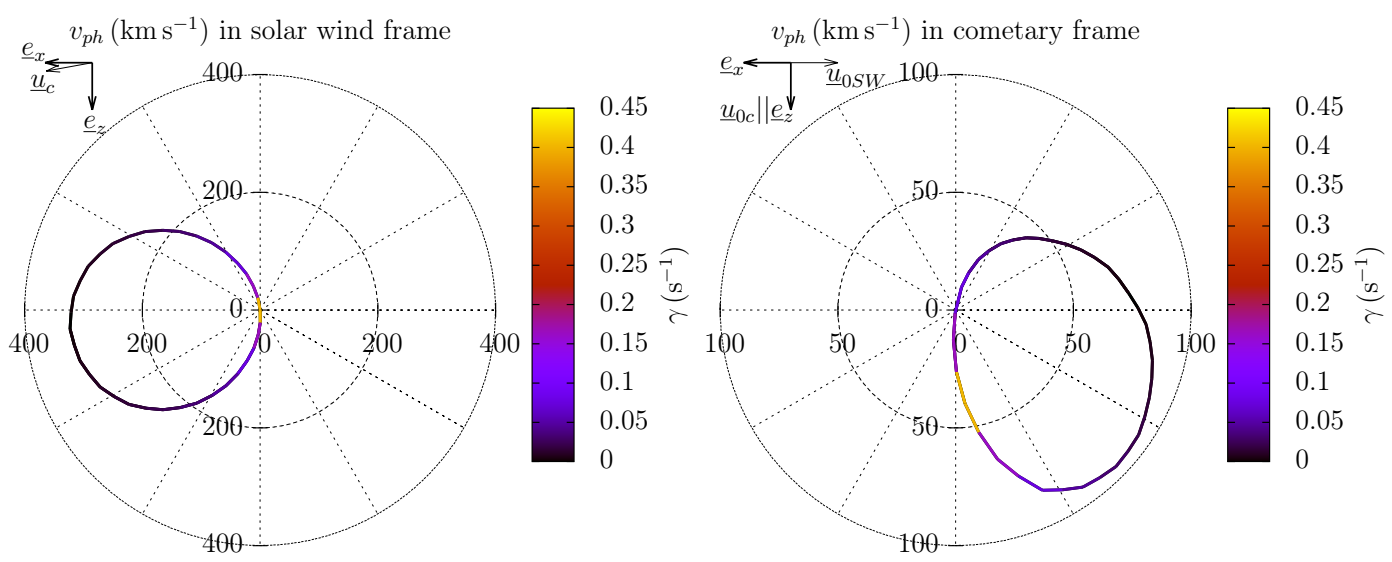

Figure 6. Wave phase velocity diagram $v_{\mathrm{ph}}=\omega_{\mathrm{c}}\left(\underline{\boldsymbol{k}}_{\max }\right) / \underline{\boldsymbol{k}}_{\max } \mid$ in SW (left) and in CSEQ (right). The velocities with colour-coded maximum growth rates $\gamma_{\mathrm{c}}\left(\underline{\boldsymbol{k}}_{\max }\right)$ are shown in the $x z$ plane corresponding to Fig. 5 (right). Additionally, the directions of characteristic velocities are indicated.

tude smaller than expected from linear models were detected by the Cluster spacecraft (Tátrallyay et al., 2008). The variation of the frequencies $f_{\max }$ in SW and CSEQ as well as the growth rate $\gamma_{c}$ are shown as function of the azimuth $\phi$ in the right panel of Fig. 5. The growth rate has narrow peaks at $\phi \approx 96^{\circ}$ and $\phi \approx 276^{\circ}$ in compliance with the propagation direction perpendicular to the ambient magnetic field and the cometary current. One also sees that the frequencies have different signs in the respective frames for most angles, i.e. the waves propagate in opposite directions due to the Doppler shift. Only close to the direction of the water ion velocity $\underline{\boldsymbol{u}}_{0 \mathrm{c}}$ the mode propagates in the same direction in both frames. An angular dependence of the frequency or growth rate is not shown in the plane spanned by $\underline{\boldsymbol{e}}_{j}$ and $\underline{\boldsymbol{e}}_{B}$, since it yields no further information. Altogether, we expect that the waves of the cometary ion mode are mainly generated within the plane perpendicular to the cometary current, where the frequencies are zero in SW.

Besides the explanation for Rosetta's measurements, our previous discussion also yields insights to the stabilization of the cometary ion mode. This mode is most unstable, i.e. each $k_{B} \in \mathbb{R}^{*}$ is unstable, if the ambient magnetic field is parallel to the wave vector $\left(\underline{\boldsymbol{k}}|| \underline{\boldsymbol{B}}_{0}\right)$ (and perpendicular to the cometary current $\left.\left(\underline{k} \perp \underline{j}_{c}\right)\right)$. On the one hand, the cometary ion mode is stabilized towards long wave lengths, where the growth rate vanishes, when a magnetic field component perpendicular to the wave vector exists. On the other hand, the cometary ion mode becomes more stable towards short wave lengths, when a cometary current component parallel to the wave vector exists. Consequently, this mode is least unstable, when the ambient magnetic field is perpendicular to and the cometary current parallel to the wave vector. However, one still finds an unstable range (Fig. 4), but with an maximum growth rate 1 order of magnitude lower than for the former cases.
Following the linear perturbation theory applied in this work (Appendix A), the dispersion relations result in unlimited growth of the modified ion-Weibel instability. However, with growing amplitudes non-linear effects kick in and a saturation of the instability is expected when $\gamma_{c, \max } \sim \omega_{\text {bounce }}$ (Davidson et al., 1972), where $\omega_{\text {bounce }}=\sqrt{e k_{\perp} u_{\mathrm{c}}|\delta \underline{\boldsymbol{B}}| / m_{\mathrm{c}}}$ is the magnetic bounce frequency. Typical amplitudes for the perturbed magnetic field of that mode of $|\delta \underline{\boldsymbol{B}}| \sim\left|\underline{\boldsymbol{B}}_{0}\right|$ are detected by Rosetta's magnetometer in the cometary environment (Richter et al., 2015) complying with the saturation condition. Therefore, a reasonable estimation for the saturation amplitude of the unstable mode is $\left|\delta \underline{\boldsymbol{B}}_{\text {sat }}\right| \sim\left|\underline{\boldsymbol{B}}_{0}\right|$.

Complementary to our discussion so far real frequencies can also be assumed, so the solutions of the dispersion relations result in complex wave numbers. In particular, we find an extremal imaginary wave number with $\left|k_{\perp}\right| \approx 6.8 \times$ $10^{-4} \mathrm{~km}^{-1}$ at $f \approx 14 \mathrm{mHz}$ corresponding to an amplification length $L_{\mathrm{Amp}}=\left|k_{\perp}^{-1}\right| \approx 1500 \mathrm{~km}$ for the modified ion-Weibel instability. This amplification length also complies with the characteristic length that can be estimated from the growth rates in Fig. 5 (left) and the solar wind velocity as group velocity yielding $L_{\mathrm{char}}=u_{0 \mathrm{SW}} / \gamma_{\mathrm{c}} \approx 1000-1700 \mathrm{~km} \approx L_{\mathrm{Amp}}$. Hence, the modified ion-Weibel instability is a convective instability (Sturrock, 1958) propagated by the solar wind.

\subsection{Phase velocity and structures}

In the previous subsection we considered the phase velocity $v_{\mathrm{ph}}=\omega_{\Re}(\underline{\boldsymbol{k}}) /|\underline{\boldsymbol{k}}|$ of the cometary ion mode only indirectly. Now, the phase velocities with maximum growth rates, i.e. corresponding to the frequencies shown in Fig. 5, are discussed in the plane spanned by the solar wind and water ions (see Fig. 1) in SW and CSEQ (Fig. 6, left and right, respectively). In SW the maximum phase velocity of $v_{\text {ph, SW }} \approx 320 \mathrm{~km} \mathrm{~s}^{-1}$ is found parallel to the cometary current $\left(\phi \approx 6^{\circ}\right)$. A wave vector antiparallel to the cometary cur- 

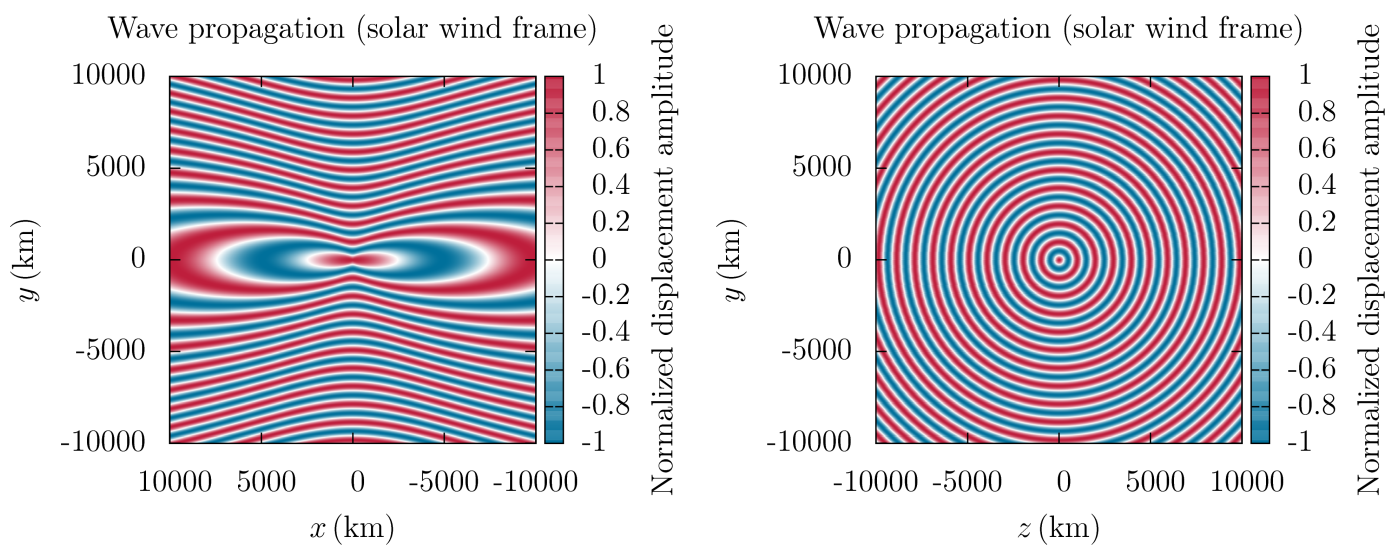

Figure 7. Illustration of the phase structure in SW. The wave $\Psi$ Eq. (19) with $\underline{\boldsymbol{k}} \perp \underline{\boldsymbol{j}}_{\mathrm{c}}$ and $|\underline{\boldsymbol{k}}|=k_{0}=6.4 \times 10^{-3} \mathrm{~km}^{-1}$ is shown in $x y$ (left) and $z y$ (right) planes.

rent yields the same velocity, but with negative sign, i.e. the propagation is still parallel to the cometary current, since the waves are carried by the water ion beam. However, the wave is also least unstable by the current, so this direction has the smallest maximum growth rate. The lowest phase velocity is zero perpendicular to the cometary current $\left(\phi \approx 96^{\circ}\right)$, but has the highest maximum growth rate as already discussed. When the wave vector is changed from perpendicular to the current to parallel and/or antiparallel to the current, the maximum growth rate and phase velocity are decreasing and increasing, respectively. In CSEQ the maximum phase velocity is $v_{\text {ph, CSEQ }} \approx 95 \mathrm{~km} \mathrm{~s}^{-1}$ at $\phi \approx 141^{\circ}$ with a rather low maximum growth rate and zero at $\phi \approx 81^{\circ}$. The highest maximum growth rate at $\phi \approx 96^{\circ}$ now corresponds to a phase velocity of $v_{\text {ph, max, CSEQ }} \approx 40 \mathrm{~km} \mathrm{~s}^{-1}$. It nearly represents the water ion velocity $\underline{\boldsymbol{u}}_{0 \mathrm{c}}$, but is slightly modified by the additional electron component of the cometary current. The phase velocity diagram is not symmetric to the cometary current because of the different solar wind and water ion velocities, since the waves are mainly carried by the solar wind. Only close to the water ion velocity $\left(\phi \approx 86^{\circ}\right)$ waves can still propagate in opposite direction to the solar wind.

Another point of interest is the spatial phase structure of the predominant waves, which we first discuss in SW and then in CSEQ. As already discussed perturbations are mainly excited in the plane perpendicular to the current. As an ansatz for these perturbations we choose a wave

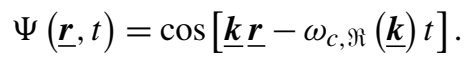

The maximum growth rates are considered by restricting the wave vectors $\underline{\boldsymbol{k}}$ to $\underline{\boldsymbol{k}} \perp \underline{\boldsymbol{j}}_{\mathrm{c}}$ and $|\underline{\boldsymbol{k}}|=k_{0}=6.4 \times 10^{-3} \mathrm{~km}^{-1}$. Furthermore, we assume only one current line in the origin as source of the wave (vectors). Then, the wave vector can be written as $\underline{\boldsymbol{k}}=\left(\begin{array}{l}k_{x} \\ k_{y} \\ k_{z}\end{array}\right)=k_{0}\left(\begin{array}{c}\cos \phi_{0} \cos \theta \\ \sin \theta \\ \sin \phi_{0} \cos \theta\end{array}\right)$

with $\phi_{0} \approx 276^{\circ}$ and the inclination

$\theta=\arctan \left(\frac{y}{x}\right) \quad$ or $\quad \theta=\arctan \left(-\frac{y}{z}\right)$,

respectively. The azimuthal angle $\phi_{0}$ is chosen instead of $96^{\circ}$ for convenience, since both yield the same growth rates (Fig. 5) and the wave vectors only point in opposite directions. The inclination $\theta$ follows from the definition of $\underline{\boldsymbol{r}}$ in spherical coordinates and the projection to the propagation plane. Note that this $\theta$ would also be called azimuth in spherical coordinates. As defined in the last subsection $\underline{\boldsymbol{k}}$ is aligned to $\underline{\boldsymbol{e}}_{x}$ for $\theta=0^{\circ}$ and is parallel to $\underline{\boldsymbol{e}}_{y}$ for $\theta=90^{\circ}$. Furthermore, the dispersion relation is $\omega_{\Re}(\underline{k})=0$ for those wave vectors (red line in Fig. 3). Hence, in the SW the wave Eq. (16) is

$$
\begin{aligned}
& \Psi(x, y, z, t) \\
& =\cos \left\{k _ { 0 } \left[x \cos \phi_{0} \cos \left(\arctan \left(\frac{y}{x}\right)\right)\right.\right. \\
& \left.\left.+y \sin \left(\arctan \left(\frac{y}{x}\right)\right)+z \sin \phi_{0} \cos \left(\arctan \left(\frac{y}{x}\right)\right)\right]\right\} \\
& =\cos \left\{k _ { 0 } \left[x \cos \phi_{0} \cos \left(\arctan \left(-\frac{y}{z}\right)\right)\right.\right. \\
& \left.\left.+y \sin \left(\arctan \left(-\frac{y}{z}\right)\right)+z \sin \phi_{0} \cos \left(\arctan \left(-\frac{y}{z}\right)\right)\right]\right\},
\end{aligned}
$$

which is shown in Fig. 7 in the $x y$ and $z y$ cross sections. The isophase structures result from the argument of the wave that is similar to the equation of an ellipse for either $x=0$ or $z=$ 0 and are independent of time, i.e. $\Psi(x, y, z, t)=\Psi(x, y, z)$ in SW. However, our equation differs from the equation of an ellipse by a geometric factor of $\left(\sqrt{x^{2}+y^{2}+z^{2}}\right)^{-1}$. The 

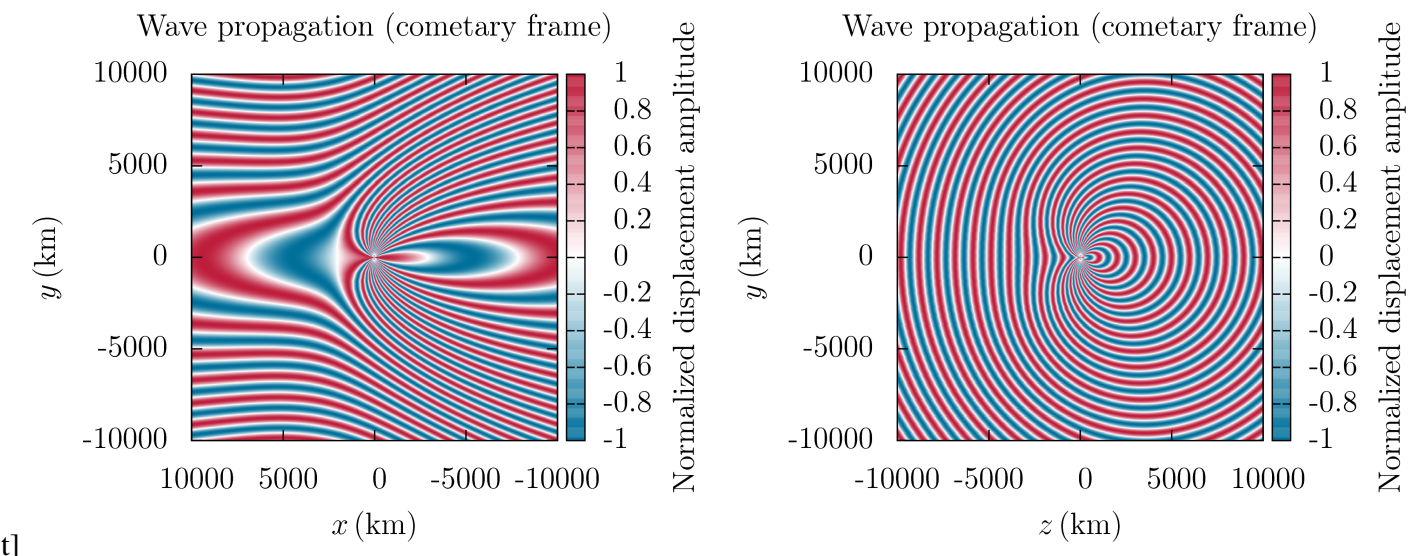

Figure 8. Wave $\Psi^{\prime}$ Eq. (20) with $t=100$ s (in CSEQ) for the same cross sections as in Fig. 7.

isophase planes are hourglass-shaped with a symmetry axis along the cometary current direction and a waist at the origin instead of a cylindric shape. On the one hand, the $z y$ cross section of this structure (Fig. 7, right) still results in nearly circular isophase lines despite the slight tilt of the hourglass. On the other hand, the isophase lines are shaped like an ellipse with waist or two merging ellipses, respectively, instead of a very eccentric ellipse in the $x y$ cross section (Fig. 7, left). Furthermore, the previously estimated wave length of $\lambda \approx 980 \mathrm{~km}$ can be clearly identified in the phase structure as the distance between two consecutive red zones in Fig. 7. One can easily see that the isophase structures of Fig. 7 correspond to a stationary striation pattern, which is typically found in the magnetic field of Weibel instabilities (Morse and Nielson, 1971), by identifying $\delta \underline{\boldsymbol{B}}=\delta \underline{\boldsymbol{B}}_{\text {sat }} \Psi(x, y, z)$.

The isophase structures of Fig. 7 are shifted by a Galilean transformation $\left(\underline{\boldsymbol{r}} \rightarrow \underline{\boldsymbol{r}}+\underline{\boldsymbol{u}}_{0 \mathrm{SW}} t\right.$ in the wave ansatz Eq. 16) to CSEQ in Fig. 8, i.e. the Doppler shift is added to the ansatz yielding the wave

$$
\begin{aligned}
& \Psi^{\prime}(x, y, z, t)=\cos \left\{k _ { 0 } \left[x \cos \phi_{0} \cos \theta\right.\right. \\
& \left.\left.+y \sin \theta+z \sin \phi_{0} \cos \theta+t u_{0 \mathrm{SW}} \cos \phi_{0} \cos \theta\right]\right\} .
\end{aligned}
$$

The phase structure is compressed by the solar wind at the sun-facing side (Fig. 8, right, $x>0$ ) and stretched at the sun-averted side (Fig. 8, right, $x<0$ ) by the Doppler shift. As the solar wind velocity is supersonic and superalfvénic the structure is similar to a Mach cone. A compression and stretching can also be seen perpendicular to the solar wind and ambient magnetic field (Fig. 8, left), since the effect of the Doppler shift is continued in this plane due to the tilt of the three-dimensional phase structure with respect to the solar wind. In particular, the isophase structures yield a fanlike shape, when we zoom in the $x y$ cross section (Fig. 9, left). Far away of the source current $(>5000 \mathrm{~km})$ the estimated wave length $\lambda \approx 980 \mathrm{~km}$ can be still identified in the phase structure, but close to the source $(<1000 \mathrm{~km})$ an apparent wave length of $\lambda \lesssim 200 \mathrm{~km}$ results from the Doppler shifted structure. This apparent wave length is in agreement with magnetometer measurements of Rosetta and its lander Philae (Richter et al., 2016). Similar fan-like structures have also been identified in simulations of the comet's plasma environment (Koenders et al., 2016). The motion of the phase structure can be identified by the frequency of the Doppler shift

$$
f=-\frac{k_{0} u_{0 \mathrm{SW}} \cos \phi_{0} \cos \theta}{2 \pi} .
$$

This term yields a frequency of $0 \leq|f| \lesssim 40 \mathrm{mHz}$ depending on the inclination $\theta$, where this frequency is positive in the anti-sunward sector and the maximum occurs for $\theta=180^{\circ}$, while it is zero parallel to the ambient magnetic field, i.e. for $\theta=90^{\circ}$ and $\theta=270^{\circ}$.

It should be noted that due to the Galilean transformation to CSEQ the phase velocity also depends on the inclination $\theta$. The phase does not move parallel to the ambient magnetic field, but with maximum phase velocity perpendicular to it. In consequence, the phase structures apparently move from the comet towards the sun. Furthermore, the isophase lines move closer together yielding the apparent wavelength clearly lower than the estimated wave length.

\subsection{Variation of background parameters}

Last, we discuss the effect of changes in the background parameters on the frequency range of the instability (red) regarding the ambient magnetic field $\left(\underline{\boldsymbol{B}}_{0}\right.$, Fig. 10), the unperturbed water ion density $\left(n_{0 \mathrm{c}}\right.$, Fig. 11$)$ and the unperturbed water ion velocity $\left(\underline{\boldsymbol{u}}_{0 \mathrm{c}}\right.$, Fig. 12$)$. While the respective quantities are varied, the other parameters remain on the values of Table 1 . The most significant changes in the frequencies result from the ambient magnetic field, where $B_{0}$ is varied from $1 \mathrm{nT}$, an estimation for the magnetic field when Rosetta arrived at the comet (Hansen et al., 2007), up to 40nT, which was measured close to perihelion (Volwerk et al., 2016). Additionally, the proton gyro-frequency $\Omega_{p}$ (blue) is shown for 

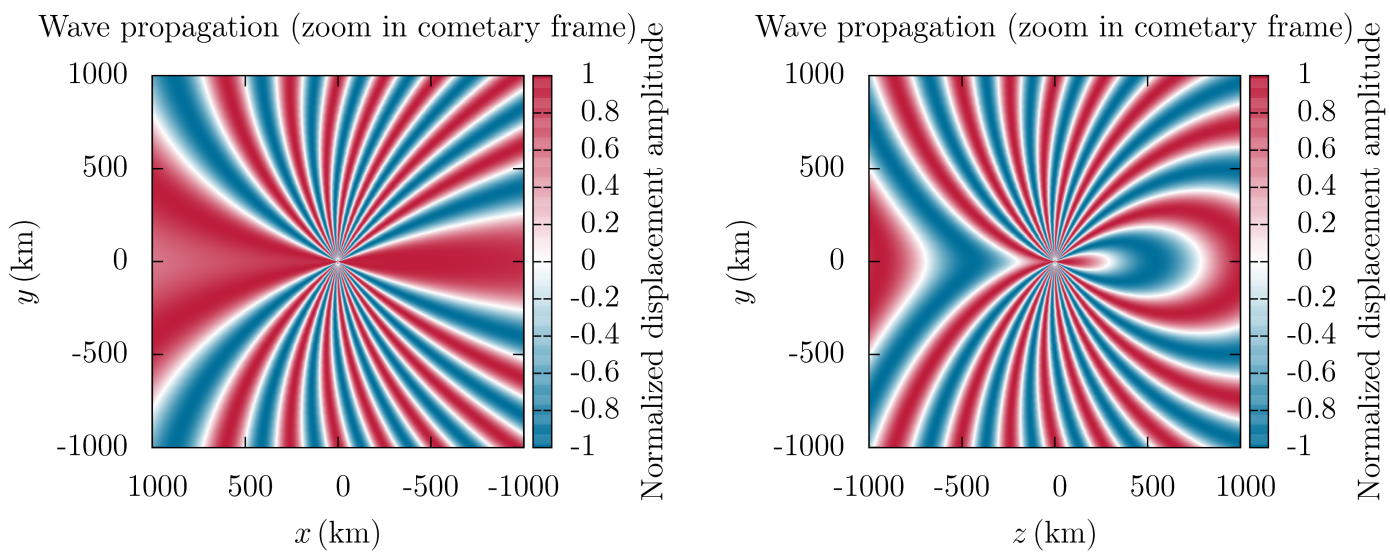

Figure 9. Zoom in Fig. 8.

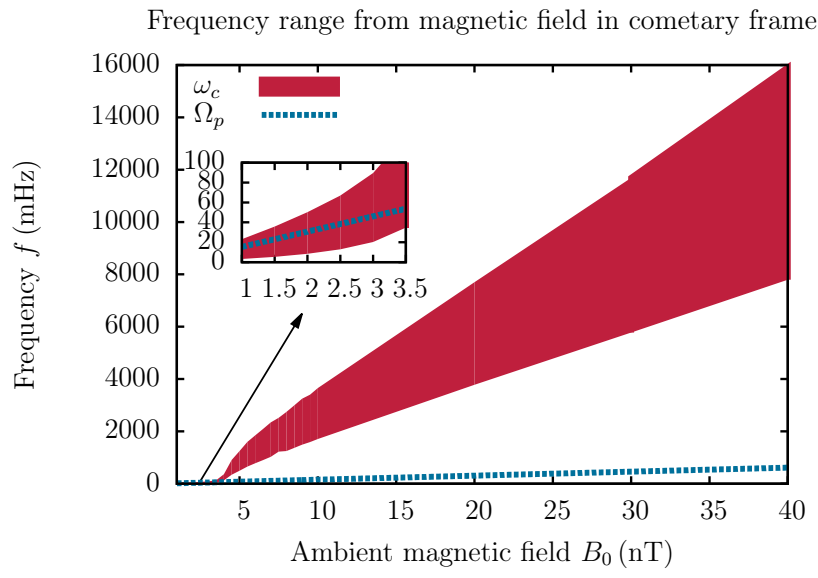

Figure 10. Variation of the frequency range of $\omega_{\mathrm{c}}$ (red) regarding the ambient magnetic field $B_{0}$ for wave propagation close to the direction with maximum growth rate $\left(86^{\circ} \lesssim \phi \lesssim 106^{\circ}\right)$ in CSEQ. The gyro-frequency of protons $\Omega_{p}$ (blue) is shown for comparison. A close-up view around the default magnetic field is shown in the inlaid panel.

comparison. Both frequencies monotonically increase with the ambient magnetic field. While $\Omega_{p}$ is proportional to $B_{0}$, a non-linear dependence is found for $\omega_{\mathrm{c}}$. Both frequencies accidentally coincide around the default field of $B_{0}=2.5 \mathrm{nT}$. For $B_{0}>10 \mathrm{nT}$ the frequencies of $\omega_{\mathrm{c}}$ clearly exceed $1 \mathrm{~Hz}$, the maximum sampling rate of Rosetta's magnetometer in normal operation mode (Richter et al., 2015). Though the magnetometer was in burst mode with a sampling rate up to $20 \mathrm{~Hz}$ around perihelion, where these fields arise, magnetic disturbances by the spacecraft are significant in the domain of a few $\mathrm{Hz}$ (Richter et al., 2016). Thus, it is unlikely to detect those waves properly close to perihelion. Furthermore, the assumptions of a homogeneous ambient magnetic field and a cometary ion beam could be less appropriate with respect
Frequency range from water ion density in cometary frame

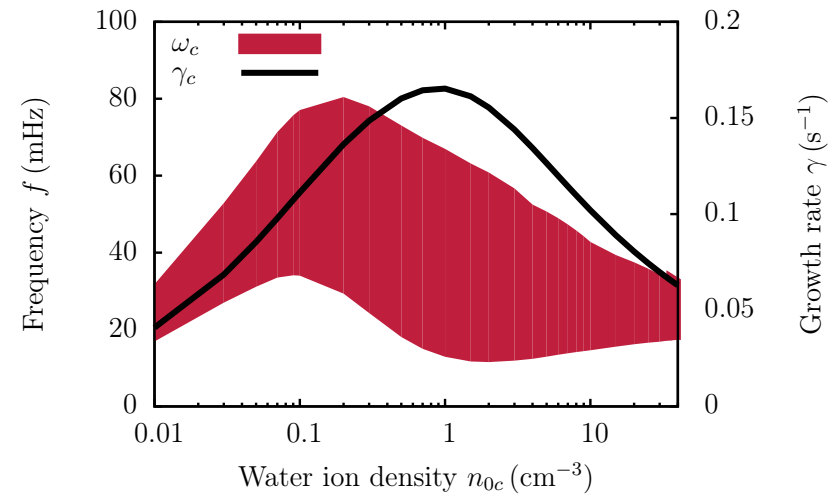

Figure 11. Variation of the frequency range of $\omega_{0}$ regarding the water ion density $n_{0 \mathrm{c}}$ analogous to Fig. 10.

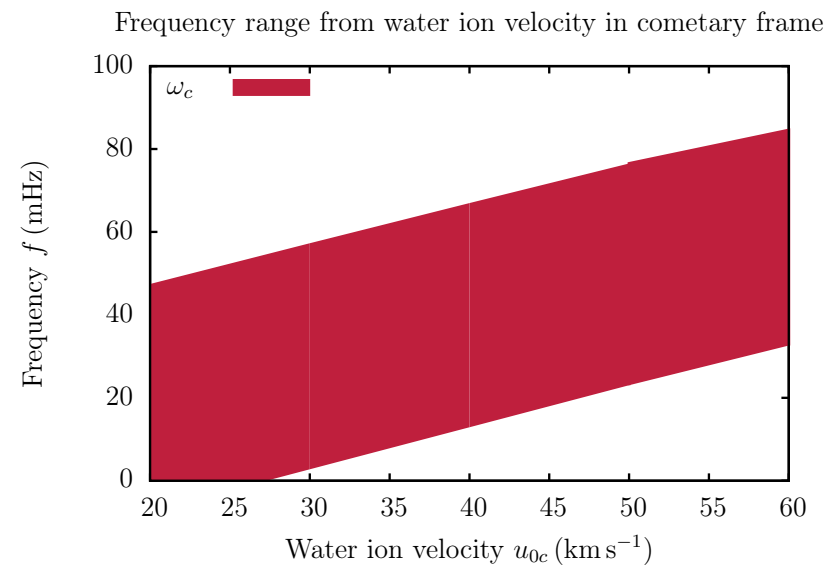

Figure 12. Variation of the frequency range of $\omega_{\mathrm{c}}$ regarding the water ion velocity $u_{0 \mathrm{c}}$ analogous to Fig. 10 .

to such high fields and gyration effects may become relevant (Volwerk et al., 2016). 
The water ion density is varied from $n_{0 \mathrm{c}}=0.01 \mathrm{~cm}^{-3}$ to $n_{0 \mathrm{c}}=40 \mathrm{~cm}^{-3}$ in Fig. 11. Besides the frequency (red), the growth rate (black) is shown, too. Both quantities have a maximum, but for different densities. The former one is found at $n_{0 \mathrm{c}} \approx 0.2 \mathrm{~cm}^{-3}$ and the latter one at $n_{0 \mathrm{c}} \approx 1 \mathrm{~cm}^{-3}$. Thus, it is most unstable when the cometary ions and protons have about the same densities. For $n_{0 \mathrm{c}} \rightarrow 0$ the frequencies and growth rate vanish as expected from Eq. (B13), while a configuration as discussed by Chang et al. (1990) is approached for $n_{0 \mathrm{p}} / n_{0 \mathrm{c}} \rightarrow 0$.

Last, the variation of the cometary ion velocity from $u_{0 \mathrm{c}}=$ $20 \mathrm{~km} \mathrm{~s}^{-1}$ to $u_{0 \mathrm{c}}=60 \mathrm{~km} \mathrm{~s}^{-1}$ is shown in Fig. 12. One can see that the frequencies (red) are nearly proportional to the velocity and the lower boundary is even below zero for $u_{0 \mathrm{c}} \lesssim 25 \mathrm{~km} \mathrm{~s}^{-1}$. However, the frequencies and the wave vector are nearly constant in SW, so this variation solely results from changes of the angle between the cometary current and the solar wind. The transition from positive to negative frequencies means a change of the propagation direction due to the Doppler effect. For a vanishing cometary ion velocity the Doppler effect is zero and the dispersion relation mainly corresponds to the results in TSW (Fig. 3).

\section{Conclusions}

In this paper we have derived a model for the new type of low-frequency waves recently detected at 67P/ChuryumovGerasimenko. Our model results well agree with measurements of the Rosetta spacecraft in the environment of the comet (Richter et al., 2015). As ansatz we choose a homogeneous, cold plasma composed of magnetized solar wind protons, magnetized electrons and a beam of unmagnetized water ions, which launch an electrical current. This configuration drives a modified ion-Weibel instability (Chang et al., 1990) that predominantly grows perpendicular to the current. The instability is connected to a frequency of about $f \approx 40 \mathrm{mHz}$ for the new water ion mode in a cometary rest frame in compliance with Rosetta's observation. Furthermore, a maximum wave length of $\lambda \approx 980 \mathrm{~km}$ is estimated. The waves propagate with phase velocities of about $v_{\mathrm{ph}} \approx 40 \mathrm{~km} \mathrm{~s}^{-1}$ perpendicular to the ambient magnetic field and the cometary current. Moreover, the superposition of the strongest growing waves results in a fan-like phase structure close to the comet that yields wave length $\lambda<200 \mathrm{~km}$ with an apparent phase motion from the comet to the sun. Last, we discussed the dependence of the frequencies on background parameters. It was shown that the frequencies of the water ion mode only coincide with the proton gyro-frequency for small ambient magnetic fields $\underline{\boldsymbol{B}}_{0} \sim 1 \mathrm{nT}$, but with different dependence on the fields, and they are clearly distinguished for stronger fields. The maxima of the growth rate and frequencies result from different water ion densities. While the water ion mode is most unstable for $n_{0 \mathrm{c}} \approx n_{0 \mathrm{p}}$, the highest frequencies are found for $n_{0 \mathrm{c}} \ll n_{0 \mathrm{p}}$. The effect of the water ion velocity on the frequencies is mainly due to variations of the cometary current direction. If the current is antiparallel to the solar wind, the Doppler effect and thus the frequencies vanish for the strongest growing waves.

\section{Data availability}

The raw data sets underlying the figures are available as supplementary material to the paper. The data are obtained as numerical solutions of the dispersion relations resulting from Eq. (12). 


\section{Appendix A: General dispersion relation}

We choose as a general ansatz a cold MHD-plasma with multiple plasma species $\alpha$. Thus, the system of equations is described by the continuity equation

$0=\partial_{t} n_{\alpha}+\partial_{\underline{x}}\left(n_{\alpha} \underline{\boldsymbol{u}}_{\alpha}\right)$,

the momentum equation

$0=\partial_{t} \underline{\boldsymbol{u}}_{\alpha}+\left(\underline{\boldsymbol{u}}_{\alpha} \partial_{\underline{x}}\right) \underline{\boldsymbol{u}}_{\alpha}-\frac{q_{\alpha}}{m_{\alpha}}\left(\underline{\boldsymbol{E}}+\underline{\boldsymbol{u}}_{\alpha} \times \underline{\boldsymbol{B}}\right)$

and Maxwell's equations

$0=\partial_{\underline{x}} \underline{\boldsymbol{B}}$,

$\frac{\rho_{\mathrm{c}}}{\varepsilon_{0}}=\partial_{\underline{x}} \underline{\boldsymbol{E}}$,

$\partial_{\underline{x}} \times \underline{\boldsymbol{E}}=-\partial_{t} \underline{\boldsymbol{B}}$,

$\partial_{\underline{x}} \times \underline{\boldsymbol{B}}=\frac{1}{c^{2}} \partial_{t} \underline{\boldsymbol{E}}+\mu_{0} \underline{\boldsymbol{j}}$,

with $n_{\alpha}, \underline{\boldsymbol{u}}_{\alpha}, q_{\alpha}$ and $m_{\alpha}$ for the number density, the bulk velocity, the charge and the mass of the plasma species $\alpha$, respectively. The electric and magnetic fields as well as the permittivity, permeability and speed of light in vacuum are denoted by $\underline{\boldsymbol{E}}$ and $\underline{\boldsymbol{B}}, \varepsilon_{0}, \mu_{0}$ and $c$, respectively. The charge and current densities are defined by

$$
\begin{aligned}
& \rho_{\mathrm{c}}=\sum_{\alpha} q_{\alpha} n_{\alpha}, \\
& \underline{\boldsymbol{j}}=\sum_{\alpha} q_{\alpha} n_{\alpha} \underline{\boldsymbol{u}}_{\alpha} .
\end{aligned}
$$

Now a first order perturbation of the plasma quantities is assumed, e.g. $n_{\alpha}=n_{0 \alpha}+\delta n_{\alpha}$. In particular, we initially allow all unperturbed quantities, which are constant and denoted by index 0 , to be non-vanishing $\left(n_{0 \alpha} \neq 0\right)$. The unperturbed charge and current densities usually have to vanish for the existence of a steady state. The first one is automatically satisfied, since the unperturbed electron density $n_{0 \mathrm{e}}$ is given by $n_{0 \mathrm{e}}=n_{0 \mathrm{p}}+n_{0 \mathrm{c}}$. The latter one does not vanish in general. But if the unperturbed current density is small, i.e. $j_{0} \mu_{0} L / B \ll 1$, a steady state can still be expected. Assuming the values of Table 1 for the current density, the size of the comet P67/Churyumov-Gerasimenko for the length scale $(L \sim 1 \mathrm{~km})$ and $B \sim 1 \mathrm{nT}$ (Richter et al., 2015), the inequality is satisfied $\left(j_{0} \mu_{0} L / B \approx 0.08\right)$.

The perturbations of the quantities are plane waves, e.g.

$\delta n_{\alpha} \propto e^{i(\underline{\underline{k}} \underline{\boldsymbol{x}}-\omega t)}$

and analogous for the other quantities, with the wave vector $\underline{\boldsymbol{k}}$ and the angular frequency $\omega$. Then, Ampere's law Eq. (A6) with Faraday's law Eq. (A5) yields

$\left[\frac{c^{2} k^{2}}{\omega^{2}}\left(\frac{k_{i} k_{l}}{k^{2}}-\delta_{i l}\right)+\delta_{i l}\right] \delta E_{l}=-\frac{i}{\varepsilon_{0} \omega}\left(j_{0 i}+\delta j_{i}\right)$, with indices $i, l \in\{j, B, \perp\}, k^{2}=|\boldsymbol{k}|^{2}$ and the delta function $\delta_{i l}$. These indices are connected to an orthonormal basis $\left\{\underline{\boldsymbol{e}}_{j}, \underline{\boldsymbol{e}}_{B}, \underline{\boldsymbol{e}}_{\perp}\right\}$ with $\underline{\boldsymbol{e}}_{B}$ parallel to an ambient magnetic field $\underline{\boldsymbol{B}}_{0}, \underline{\boldsymbol{e}}_{j}$ parallel to a current density $\underline{\boldsymbol{j}}_{0}$ and $\underline{\boldsymbol{e}}_{\perp}$ perpendicular to the field as well as the current completing this righthanded system. Next, the perturbation of the current density is derived from Eqs. (A1) and (A2)

$\delta j_{i}=\sum_{\alpha} \sigma_{i l \alpha} \delta E_{l}$,

with wave conductivity tensor $\sigma_{i l \alpha}$ of species $\alpha$. The conductivity tensor is not shown, but can be easily read off of the following tensor. Equation (A10) is rewritten with the dispersion tensor $D_{i l}$

$D_{i l} \delta E_{l}=\left[\frac{c^{2} k^{2}}{\omega^{2}}\left(\frac{k_{i} k_{l}}{k^{2}}-\delta_{i l}\right)+\epsilon_{i l}\right] \delta E_{l}=\Psi_{i}(\omega, \underline{\boldsymbol{k}})$,

with dielectric tensor $\epsilon_{i l}=\delta_{i l}+i \sum_{\alpha} \sigma_{i l \alpha} /\left(\omega \varepsilon_{0}\right)$ and $\Psi_{i}(\omega, \boldsymbol{k})$, which depends on plasma quantities of the steady state, but not on perturbed plasma quantities. The expressions for the components of $D_{i j}$ are

$D_{j j}=1-\frac{c^{2}\left(k_{B}^{2}+k_{\perp}^{2}\right)}{\omega^{2}}$

$-\sum_{\alpha} \frac{\omega_{\alpha}^{2}}{\omega^{2}}\left[\frac{\left(\omega-\underline{\boldsymbol{k}}_{0 \alpha}+k_{j} u_{0 j \alpha}\right)^{2}+k_{\perp}^{2} u_{0 j \alpha}^{2}}{\left(\omega-\underline{\boldsymbol{k}} \underline{\boldsymbol{u}}_{0 \alpha}\right)^{2}-\Omega_{\alpha}^{2}}+\frac{k_{B}^{2} u_{0 j \alpha}^{2}}{\left(\omega-\underline{\boldsymbol{k}} \underline{\boldsymbol{u}}_{0 \alpha}\right)^{2}}\right]$

$D_{B B}=1-\frac{c^{2}\left(k_{j}^{2}+k_{\perp}^{2}\right)}{\omega^{2}}$

$-\sum_{\alpha} \frac{\omega_{\alpha}^{2}}{\omega^{2}}\left[\frac{\left(\omega-\underline{\boldsymbol{k}} \underline{\boldsymbol{u}}_{0 \alpha}+k_{B} u_{0 B \alpha}\right)^{2}}{\left(\omega-\underline{\boldsymbol{k}} \underline{\boldsymbol{u}}_{0 \alpha}\right)^{2}}+\frac{\left(k_{j}^{2}+k_{\perp}^{2}\right) u_{0 B \alpha}^{2}}{\left(\omega-\underline{\boldsymbol{k}} \underline{\boldsymbol{u}}_{0 \alpha}\right)^{2}-\Omega_{\alpha}^{2}}\right]$

$D_{\perp \perp}=1-\frac{c^{2}\left(k_{j}^{2}+k_{B}^{2}\right)}{\omega^{2}}$

$-\sum_{\alpha} \frac{\omega_{\alpha}^{2}}{\omega^{2}}\left[\frac{\left(\omega-\underline{\boldsymbol{k}} \underline{\boldsymbol{u}}_{0 \alpha}+k_{\perp} u_{0 \perp \alpha}\right)^{2}+k_{j}^{2} u_{0 \perp \alpha}^{2}}{\left(\omega-\underline{\boldsymbol{k}}_{0 \alpha}\right)^{2}-\Omega_{\alpha}^{2}}+\frac{k_{B}^{2} u_{0 \perp \alpha}^{2}}{\left(\omega-\underline{\boldsymbol{k}} \underline{\boldsymbol{u}}_{0 \alpha}\right)^{2}}\right]$

$D_{j B}=\frac{c^{2} k_{j} k_{B}}{\omega^{2}}-\sum_{\alpha} \frac{\omega_{\alpha}^{2}}{\omega^{2}}$

$\left[\frac{\left(\omega-\underline{\boldsymbol{k}} \underline{\boldsymbol{u}}_{0 \alpha}\right) k_{j}+\left(k_{j}^{2}+k_{\perp}^{2}\right) u_{0 j \alpha}}{\left(\omega-\underline{\boldsymbol{k}} \underline{\boldsymbol{u}}_{0 \alpha}\right)^{2}-\Omega_{\alpha}^{2}} u_{0 B \alpha}\right.$

$+\frac{\left(\omega-k_{j} u_{0 j \alpha}-k_{\perp} u_{0 \perp \alpha}\right) k_{B} u_{0 j \alpha}}{\left(\omega-\underline{\boldsymbol{k}} \underline{\boldsymbol{u}}_{0 \alpha}\right)^{2}}$

$\left.+\frac{i \Omega_{\alpha} k_{\perp} u_{0 B \alpha}}{\left(\omega-\underline{k} \underline{\boldsymbol{u}}_{0 \alpha}\right)^{2}-\Omega_{\alpha}^{2}}\right]$ 
$D_{j \perp}=\frac{c^{2} k_{j} k_{\perp}}{\omega^{2}}-\sum_{\alpha} \frac{\omega_{\alpha}^{2}}{\omega^{2}}$

$\left[\frac{\left(\omega-\underline{\boldsymbol{k}}_{0 \alpha}\right)\left(k_{j} u_{0 \perp \alpha}+k_{\perp} u_{0 j \alpha}\right)+\left(k_{j}^{2}+k_{\perp}^{2}\right) u_{0 \perp \alpha} u_{0 j \alpha}}{\left(\omega-\underline{\boldsymbol{k}} \underline{\boldsymbol{u}}_{0 \alpha}\right)^{2}-\Omega_{\alpha}^{2}}\right.$

$\left.+\frac{k_{B}^{2} u_{0 j \alpha} u_{0 \perp \alpha}}{\left(\omega-\underline{\boldsymbol{k}} \underline{\boldsymbol{u}}_{0 \alpha}\right)^{2}}+\frac{i \Omega_{\alpha}\left(\omega-k_{B} u_{0 B \alpha}\right)}{\left(\omega-\underline{\boldsymbol{k}} \underline{\boldsymbol{u}}_{0 \alpha}\right)^{2}-\Omega_{\alpha}^{2}}\right]$

$D_{B \perp}=\frac{c^{2} k_{B} k_{\perp}}{\omega^{2}}-\sum_{\alpha} \frac{\omega_{\alpha}^{2}}{\omega^{2}}$

$\left[\frac{\left(\omega-\underline{\boldsymbol{k}}_{0 \alpha}\right) k_{\perp}+\left(k_{j}^{2}+k_{\perp}^{2}\right) u_{0 \perp \alpha}}{\left(\omega-\underline{\boldsymbol{k}} \underline{\boldsymbol{u}}_{0 \alpha}\right)^{2}-\Omega_{\alpha}^{2}} u_{0 B \alpha}\right.$

$+\frac{\left(\omega-k_{j} u_{0 j \alpha}-k_{\perp} u_{0 \perp \alpha}\right) k_{B} u_{0 \perp \alpha}}{\left(\omega-\underline{\boldsymbol{u}}_{0 \alpha}\right)^{2}}$

$\left.+\frac{i \Omega_{\alpha} k_{j} u_{0 B \alpha}}{\left(\omega-\underline{\boldsymbol{k}} \underline{\boldsymbol{u}}_{0 \alpha}\right)^{2}-\Omega_{\alpha}^{2}}\right]$

$D_{B j}=D_{j B}^{*} \quad D_{\perp j}=D_{j \perp}^{*} \quad D_{\perp B}=D_{B \perp}^{*}$, with plasma frequencies $\omega_{\alpha}=\sqrt{q_{\alpha}^{2} n_{0 \alpha} /\left(\varepsilon_{0} m_{\alpha}\right)}$ and gyrofrequencies $\Omega_{\alpha}=q_{\alpha}\left|\underline{\boldsymbol{B}}_{0}\right| / m_{\alpha}$ of the respective plasma species $\alpha$. Note that the gyro-frequency can be signed by the charge. Following, we are interested in waves, which propagate in plasmas independent of the steady state, i.e. which satisfy the dispersion relation

$\operatorname{det}\left(D_{i j}\right)=0$. 


\section{Appendix B: Cut-off and resonance frequencies}

We start the further examination with waves that propagate perpendicular to the magnetic field, so the dispersion relation Eq. (12) resulting from Eqs. (5-11) is reduced to

$$
\left(D_{j j} D_{\perp \perp}-D_{j \perp} D_{j \perp}^{*}\right) D_{B B}=0 .
$$

Hence, $D_{B B}$ is decoupled and the mode is given by

$\omega= \pm \sqrt{c^{2}\left(k_{j}^{2}+k_{\perp}^{2}\right)+\omega_{e}^{2}+\omega_{p}^{2}+\omega_{\mathrm{c}}^{2}}$,

which represents a modified O-mode. However, we are interested in low-frequency waves $\left(\omega^{2} \ll \omega_{e}^{2}\right)$ and this highfrequency mode is not further discussed. For low-frequency waves the general dispersion relation can be approximated by

$$
\begin{aligned}
0 & =\left(D_{j j} D_{\perp \perp}-D_{j \perp} D_{j \perp}^{*}\right) D_{B B} \\
& +\left(D_{j \perp}+D_{j \perp}^{*}\right) D_{B \perp} D_{j B}-D_{\perp \perp} D_{j B}^{2}-D_{j j} D_{B \perp}^{2} \\
& \approx\left(D_{j j} D_{\perp \perp}-D_{j \perp} D_{j \perp}^{*}\right) D_{B B}
\end{aligned}
$$

and decoupling of $D_{B B}$ can be again assumed, so only the dispersion relation

$0=\left|\left(\begin{array}{cc}D_{j j} & D_{j \perp} \\ D_{j \perp}^{*} & D_{\perp \perp}\end{array}\right)\right|$

has to be solved. This dispersion tensor can be diagonalized by an unitary transformation yielding

$0=\left|\left(\begin{array}{cc}\sigma_{1} & 0 \\ 0 & \sigma_{2}\end{array}\right)\right|$,

with

$$
\sigma_{1}=D_{j j}+2 \frac{\left|D_{j \perp}\right|^{2}}{\left(D_{j j}-D_{\perp \perp}\right)\left(1 \pm \sqrt{1+4 \frac{\left|D_{j \perp}\right|^{2}}{\left(D_{j j}-D_{\perp \perp}\right)^{2}}}\right)}
$$

and

$$
\sigma_{2}=D_{\perp \perp}-2 \frac{\left|D_{j \perp}\right|^{2}}{\left(D_{j j}-D_{\perp \perp}\right)\left(1 \pm \sqrt{1+4 \frac{\left|D_{j \perp}\right|^{2}}{\left(D_{j j}-D_{\perp \perp}\right)^{2}}}\right)} .
$$

We have already seen that a modified O-mode Eq. (B2) can be identified in the dispersion relation. Therefore, it is also expected to find expressions for the other modes, which can be characterized by their cut-off and resonance frequencies and merge with the well-known terms in the limit of $n_{0 \mathrm{c}} \rightarrow 0$. We begin with wave propagation parallel to the magnetic field $\left(k_{j}=k_{\perp}=0\right)$. Since the low-frequency domain is studied, the effect of the displacement current in Eqs. (5-7) can also be neglected and the dispersion relations Eq. (B5) are

$$
\begin{gathered}
0=\frac{c^{2} k_{B}^{2}}{\omega^{2}}+\frac{\omega_{e}^{2}}{\omega^{2}-\Omega_{e}^{2}}+\frac{\omega_{p}^{2}}{\omega^{2}-\Omega_{p}^{2}}+\frac{\omega_{\mathrm{c}}^{2}}{\omega^{2}} \\
-\frac{\frac{2 \omega^{2}}{\omega_{\mathrm{c}}^{2} k_{B}^{2} u_{\mathrm{c}}^{2}}\left(\frac{\Omega_{e} \omega_{e}^{2}}{\omega^{2}-\Omega_{e}^{2}}+\frac{\Omega_{p} \omega_{p}^{2}}{\omega^{2}-\Omega_{p}^{2}}\right)^{2}}{1 \pm \sqrt{1+4 \frac{\omega^{6}}{\omega_{\mathrm{c}}^{4} k_{B}^{4} u_{\mathrm{c}}^{4}}\left(\frac{\Omega_{e} \omega_{e}^{2}}{\omega^{2}-\Omega_{e}^{2}}+\frac{\Omega_{p} \omega_{p}^{2}}{\omega^{2}-\Omega_{p}^{2}}\right)^{2}}}
\end{gathered}
$$

and

$$
\begin{gathered}
0=\frac{c^{2} k_{B}^{2}}{\omega^{2}}+\frac{\omega_{e}^{2}}{\omega^{2}-\Omega_{e}^{2}}+\frac{\omega_{p}^{2}}{\omega^{2}-\Omega_{p}^{2}}+\frac{\omega_{\mathrm{c}}^{2}}{\omega^{4}}\left(\omega^{2}+k_{B}^{2} u_{\mathrm{c}}^{2}\right) \\
+\frac{\frac{2 \omega^{2}}{\omega_{\mathrm{c}}^{2} k_{B}^{2} u_{\mathrm{c}}^{2}}\left(\frac{\Omega_{e} \omega_{e}^{2}}{\omega^{2}-\Omega_{e}^{2}}+\frac{\Omega_{p} \omega_{p}^{2}}{\omega^{2}-\Omega_{p}^{2}}\right)^{2}}{1 \pm \sqrt{1+4 \frac{\omega^{6}}{\omega_{\mathrm{c}}^{4} k_{B}^{4} u_{\mathrm{c}}^{4}}\left(\frac{\Omega_{e} \omega_{e}^{2}}{\omega^{2}-\Omega_{e}^{2}}+\frac{\Omega_{p} \omega_{p}^{2}}{\omega^{2}-\Omega_{p}^{2}}\right)^{2}}} .
\end{gathered}
$$

The last term in both equations vanishes in the resonance $k_{B} \rightarrow \infty$ and it remains

$\frac{c^{2} k_{B}^{2}}{\omega^{2}}=-\left(\frac{\omega_{e}^{2}}{\omega^{2}-\Omega_{e}^{2}}+\frac{\omega_{p}^{2}}{\omega^{2}-\Omega_{p}^{2}}+\frac{\omega_{\mathrm{c}}^{2}}{\omega^{2}}\right)$

and

$\frac{c^{2} k_{B}^{2}}{\omega^{2}}=-\frac{1}{1+\frac{\omega_{\mathrm{c}}^{2}}{\omega^{2}} \frac{u_{\mathrm{c}}^{2}}{c^{2}}}\left(\frac{\omega_{e}^{2}}{\omega^{2}-\Omega_{e}^{2}}+\frac{\omega_{p}^{2}}{\omega^{2}-\Omega_{p}^{2}}+\frac{\omega_{\mathrm{c}}^{2}}{\omega^{2}}\right)$

with resonance frequencies $\omega_{R}$, res $= \pm \Omega_{e}, \omega_{L \text {, res }}= \pm \Omega_{p}$ and $\omega_{c \text {, res }}= \pm i \omega_{\mathrm{c}} u_{\mathrm{c}} / c$. The first two resonance frequencies correspond to the R- and L-modes, respectively, while the last resonance appears due to the motion of the additional cometary ions and is pure imaginary, i.e. the last mode does not propagate along the magnetic field.

Next, the resonance frequencies for propagation perpendicular to the magnetic field and the cometary current $\left(k_{j}=\right.$ $k_{B}=0$ ) are discussed. We get the dispersion relations

$$
\begin{aligned}
& 0=\left(1+\frac{\omega_{\mathrm{c}}^{2}}{\omega^{2}} \frac{u_{\mathrm{c}}^{2}}{c^{2}}\right) \\
& \left(\frac{\omega_{e}^{2}}{\omega^{2}-\Omega_{e}^{2}}+\frac{\omega_{p}^{2}}{\omega^{2}-\Omega_{p}^{2}}\right)+\frac{\omega_{\mathrm{c}}^{2}}{\omega^{2}}
\end{aligned}
$$

and

$$
\begin{aligned}
\frac{c^{2} k_{\perp}^{2}}{\omega^{2}} & =-\frac{1}{1+\frac{\omega_{\mathrm{c}}^{2}}{\omega^{2}} \frac{u_{\mathrm{c}}^{2}}{c^{2}}} \\
& \left(\frac{\omega_{e}^{2}}{\omega^{2}-\Omega_{e}^{2}}+\frac{\omega_{p}^{2}}{\omega^{2}-\Omega_{p}^{2}}+\frac{\omega_{\mathrm{c}}^{2}}{\omega^{2}} \frac{1+2 \frac{\omega_{\mathrm{c}}^{2}}{\omega^{2}} \frac{u_{\mathrm{c}}^{2}}{c^{2}}}{1+\frac{\omega_{\mathrm{c}}^{2}}{\omega^{2}} \frac{u_{\mathrm{c}}^{2}}{c^{2}}}\right)
\end{aligned}
$$


in the resonance $k_{\perp} \rightarrow \infty$ analogous to Eqs. (B10) and (B11). Two resonance frequencies

$\omega_{X, \text { res }}=$

$\pm \sqrt{\frac{\Omega_{e}^{2}\left(\omega_{p}^{2}+\omega_{\mathrm{c}}^{2}\right)+\Omega_{p}^{2}\left(\omega_{e}^{2}+\omega_{\mathrm{c}}^{2}\right)-\omega_{\mathrm{c}}^{2} \frac{u_{\mathrm{c}}^{2}}{c^{2}}\left(\omega_{e}^{2}+\omega_{p}^{2}\right)}{\omega_{e}^{2}+\omega_{p}^{2}+\omega_{\mathrm{c}}^{2}}}$

and

$\omega_{c, \text { res }}=$

$\pm \omega_{\mathrm{c}} \sqrt{\frac{\Omega_{e}^{2} \Omega_{p}^{2}-\frac{u_{\mathrm{c}}^{2}}{c^{2}}\left(\omega_{e}^{2} \Omega_{p}^{2}+\omega_{p}^{2} \Omega_{e}^{2}\right)}{\Omega_{e}^{2}\left(\omega_{p}^{2}+\omega_{\mathrm{c}}^{2}\right)+\Omega_{p}^{2}\left(\omega_{e}^{2}+\omega_{\mathrm{c}}^{2}\right)-\omega_{\mathrm{c}}^{2} \frac{u_{\mathrm{c}}^{2}}{c^{2}}\left(\omega_{e}^{2}+\omega_{p}^{2}\right)}}$

result from Eq. (B12). The first resonance frequency approaches the lower-hybrid frequency

$\omega_{X, \text { res }} \rightarrow \pm \sqrt{\frac{\Omega_{e}^{2} \omega_{p}^{2}+\Omega_{p}^{2} \omega_{e}^{2}}{\omega_{e}^{2}+\omega_{p}^{2}}}$

in the limit $n_{0 \mathrm{c}} \rightarrow 0$, so this mode corresponds to a modified $\mathrm{X}$-mode. The second resonance frequency belongs to a new mode regarding the cometary ions. This frequency is again imaginary in the comet's environment (Table 1). The previous resonances parallel to the magnetic field would result from Eq. (B13), but are prohibited by Eq. (B12).

Last, the resonance frequencies for propagation parallel to the cometary current $\left(k_{B}=k_{\perp}=0\right)$ are studied. The resonance frequencies can easier be found from the original dispersion relation Eq. (B4) than from the diagonalized one. It yields $\frac{c^{2} k_{j}^{2}}{\omega^{2}}=$

$$
-\frac{\left(\frac{\omega_{e}^{2}}{\omega^{2}-\Omega_{e}^{2}}+\frac{\omega_{p}^{2}}{\omega^{2}-\Omega_{p}^{2}}+\frac{\omega_{c}^{2}}{\omega^{2}}\right)\left(\frac{\omega_{e}^{2}}{\omega^{2}-\Omega_{e}^{2}}+\frac{\omega_{p}^{2}}{\omega^{2}-\Omega_{p}^{2}}+\frac{\omega_{c}^{2}}{\left(\omega-k_{j} u_{\mathrm{c}}\right)^{2}}\right)-\frac{1}{\omega^{2}}\left(\frac{\Omega_{e} \omega_{e}^{2}}{\omega^{2}-\Omega_{e}^{2}}+\frac{\Omega_{e} \omega_{p}^{2}}{\omega^{2}-\Omega_{p}^{2}}\right)^{2}}{\frac{\omega_{e}^{2}}{\omega^{2}-\Omega_{e}^{2}}+\frac{\omega_{p}^{2}}{\omega^{2}-\Omega_{p}^{2}}+\frac{\omega_{c}^{2}}{\left(\omega-k_{j} u_{\mathrm{c}}\right)^{2}}},
$$

where the term $\omega_{\mathrm{c}}^{2}\left(\omega-k_{j} u_{\mathrm{c}}\right)^{-2}$ vanishes in the resonance $k_{j} \rightarrow \infty$. One resonance frequency, which is the lowerhybrid frequency Eq. (B16) that again indicates a modified $\mathrm{X}$-mode, results from the root of the denominator. Other resonances do not appear in the low-frequency domain.

The last characteristic frequencies, which can be analytically derived, are the cut-off frequencies $\left(k_{j}=k_{B}=k_{\perp}=\right.$ $0)$. The dispersion relation yields

$0=\frac{\omega_{e}^{2}}{\omega\left(\omega \pm \Omega_{e}\right)}+\frac{\omega_{p}^{2}}{\omega\left(\omega \pm \Omega_{p}\right)}+\frac{\omega_{\mathrm{c}}^{2}}{\omega^{2}}$.

The corresponding cut-off frequencies are

$\omega_{c u t} \approx \mp \frac{\omega_{\mathrm{c}}^{2} \Omega_{e} \Omega_{p}}{\omega_{e}^{2} \Omega_{p}+\omega_{p}^{2} \Omega_{e}+\omega_{\mathrm{c}}^{2}\left(\Omega_{e}+\Omega_{p}\right)}$

and

$\omega_{c u t} \approx \mp \frac{\omega_{e}^{2} \Omega_{p}+\omega_{p}^{2} \Omega_{e}+\omega_{\mathrm{c}}^{2}\left(\Omega_{e}+\Omega_{p}\right)}{\omega_{e}^{2}+\omega_{p}^{2}+\omega_{\mathrm{c}}^{2}}$.

In the limit $n_{0 \mathrm{c}} \rightarrow 0$ both cut-offs approach $\omega_{c u t} \rightarrow 0$, the first obliviously due to $\omega_{\mathrm{c}} \rightarrow 0$ and the second, additionally, due to $\omega_{e}^{2} \Omega_{p} \rightarrow-\omega_{p}^{2} \Omega_{e}$ according the quasi-neutrality, as expected for each mode in a two species (electron-ion) plasma. 


\section{The Supplement related to this article is available online at doi:10.5194/angeo-34-691-2016-supplement.}

Acknowledgements. This study was financially supported by the German Bundesministerium für Wirtschaft und Energie and the Deutsches Zentrum für Luft- und Raumfahrt under grant 50QP 1401.

The topical editor, C. Owen, thanks M. V. Volwerk and one anonymous referee for help in evaluating this paper.

\section{References}

Acton, C. H.: Ancillary data services of NASA's Navigation and Ancillary Information Facility, Planet. Space Sci., 44, 65-70, doi:10.1016/0032-0633(95)00107-7, 1996.

Arnold, P. and Moore, G. D.: QCD plasma instabilities: The non-Abelian cascade, Phys. Rev. D, 73, 025006, doi:10.1103/PhysRevD.73.025006, 2006.

Baumjohann, W. and Treumann, R.: Basic Space Plasma Physics, Imperial College Press, availabe at: https://books.google.de/ books?id=e4yupcOzJxkC, 1997.

Chang, C. L., Wong, H. K., and Wu, C. S.: Electromagnetic instabilities attributed to a cross-field ion drift, Phys. Rev. Lett., 65, 1104-1107, doi:10.1103/PhysRevLett.65.1104, 1990.

Davidson, R. C., Hammer, D. A., Haber, I., and Wagner, C. E.: Nonlinear Development of Electromagnetic Instabilities in Anisotropic Plasmas, Phys. Fluids, 15, 317-333, doi:10.1063/1.1693910, 1972.

Drummond, W. E. and Rosenbluth, M. N.: Anomalous Diffusion Arising from Microinstabilities in a Plasma, Phys. Fluids, 5, 1507-1513, doi:10.1063/1.1706559, 1962.

Fried, B. D.: Mechanism for Instability of Transverse Plasma Waves, Phys. Fluids, 2, 337-337, doi:10.1063/1.1705933, 1959.

Gary, S. P.: The mirror and ion cyclotron anisotropy instabilities, J. Geophys. Res., 97, 8519-8529, doi:10.1029/92JA00299, 1992.

Gary, S. P., Montgomery, M. D., Feldman, W. C., and Forslund, D. W.: Proton temperature anisotropy instabilities in the solar wind, J. Geophys. Res., 81, 1241-1246, doi:10.1029/JA081i007p01241, 1976.

Glassmeier, K.-H. and Neubauer, F. M.: Low-frequency electromagnetic plasma waves at comet P/Grigg-Skjellerup: Overview and spectral characteristics, J. Geophys. Res., 98, 20921-20935, doi:10.1029/93JA02583, 1993.

Glassmeier, K.-H., Coates, A. J., Acuna, M. H., Goldstein, M. L., Johnstone, A. D., Neubauer, F. M., and Reme, H.: Spectral characteristics of low-frequency plasma turbulence upstream of Comet P/Halley, J. Geophys. Res., 94, 37-48, doi:10.1029/JA094iA01p00037, 1989.

Glassmeier, K.-H., Boehnhardt, H., Koschny, D., Kührt, E., and Richter, I.: The Rosetta Mission: Flying Towards the Origin of the Solar System, Space Sci. Rev., 128, 1-21, doi:10.1007/s11214-006-9140-8, 2007a.

Glassmeier, K.-H., Richter, I., Diedrich, A., Musmann, G., Auster, U., Motschmann, U., Balogh, A., Carr, C., Cupido, E., Coates, A., Rother, M., Schwingenschuh, K., Szegö, K., and Tsurutani, B.: RPC-MAG The Fluxgate Magnetometer in the
ROSETTA Plasma Consortium, Space Sci. Rev., 128, 649-670, doi:10.1007/s11214-006-9114-x, 2007b.

Hansen, K. C., Bagdonat, T., Motschmann, U., Alexander, C., Combi, M. R., Cravens, T. E., Gombosi, T. I., Jia, Y.-D., and Robertson, I. P.: The Plasma Environment of Comet 67P/Churyumov-Gerasimenko Throughout the Rosetta Main Mission, Space Sci. Rev., 128, 133-166, doi:10.1007/s11214006-9142-6, 2007.

Kindel, J. M. and Kennel, C. F.: Topside current instabilities, J. Geophys. Res., 76, 3055-3078, doi:10.1029/JA076i013p03055, 1971.

Koenders, C., Glassmeier, K.-H., Richter, I., Motschmann, U., and Rubin, M.: Revisiting cometary bow shock positions, Planet. Space Sci., 87, 85-95, doi:10.1016/j.pss.2013.08.009, 2013.

Koenders, C., Perschke, C., Goetz, C., Richter, I., Motschmann, U., and Glassmeier, K. H.: Low-frequency waves at comet 67P/Churyumov-Gerasimenko, Astron. Astrophys., doi:10.1051/0004-6361/201628803, submitted, 2016.

Lui, A. T. Y., Chang, C.-L., Mankofsky, A., Wong, H.K., and Winske, D.: A cross-field current instability for substorm expansions, J. Geophys. Res., 96, 11389-11401, doi:10.1029/91JA00892, 1991.

Milosavljević, M., Nakar, E., and Spitkovsky, A.: Steady State Electrostatic Layers from Weibel Instability in Relativistic Collisionless Shocks, Astrophys. J., 637, 765-773, doi:10.1086/498445, 2006.

Morse, R. L. and Nielson, C. W.: Numerical Simulation of the Weibel Instability in One and Two Dimensions, Phys. Fluids, 14, 830-840, doi:10.1063/1.1693518, 1971.

Motschmann, U. and Glassmeier, K.-H.: Nongyrotropic distribution of pickup ions at comet P/Grigg-Skjellerup: A possible source of wave activity, J. Geophys. Res., 98, 20977-20983, doi:10.1029/93JA02533, 1993.

Neubauer, F. M., Glassmeier, K. H., Pohl, M., Raeder, J., Acuna, M. H., Burlaga, L. F., Ness, N. F., Musmann, G., Mariani, F., Wallis, M. K., Ungstrup, E., and Schmidt, H. U.: First results from the Giotto magnetometer experiment at comet Halley, Nature, 321, 352-355, doi:10.1038/321352a0, 1986.

Nilsson, H., Stenberg Wieser, G., Behar, E., Wedlund, C. S., Gunell, H., Yamauchi, M., Lundin, R., Barabash, S., Wieser, M., Carr, C., Cupido, E., Burch, J. L., Fedorov, A., Sauvaud, J.-A., Koskinen, H., Kallio, E., Lebreton, J.-P., Eriksson, A., Edberg, N., Goldstein, R., Henri, P., Koenders, C., Mokashi, P., Nemeth, Z., Richter, I., Szego, K., Volwerk, M., Vallat, C., and Rubin, M.: Birth of a comet magnetosphere: A spring of water ions, Science, 347, 6220, doi:10.1126/science.aaa0571, 2015.

Richter, I., Koenders, C., Auster, H.-U., Frühauff, D., Götz, C., Heinisch, P., Perschke, C., Motschmann, U., Stoll, B., Altwegg, K., Burch, J., Carr, C., Cupido, E., Eriksson, A., Henri, P., Goldstein, R., Lebreton, J.-P., Mokashi, P., Nemeth, Z., Nilsson, H., Rubin, M., Szegö, K., Tsurutani, B. T., Vallat, C., Volwerk, M., and Glassmeier, K.-H.: Observation of a new type of low-frequency waves at comet 67P/Churyumov-Gerasimenko, Ann. Geophys., 33, 1031-1036, doi:10.5194/angeo-33-10312015, 2015.

Richter, I., Auster, H.-U., Berghofer, G., Carr, C., Cupido, E., Fornaçon, K.-H., Goetz, C., Heinisch, P., Koenders, C., Stoll, B., Tsurutani, B. T., Vallat, C., Volwerk, M., and Glassmeier, K.-H.: Two-point observations of low-frequency waves 
at 67P/Churyumov-Gerasimenko during the descent of PHILAE: comparison of RPCMAG and ROMAP, Ann. Geophys., 34, 609622, doi:10.5194/angeo-34-609-2016, 2016.

Rubin, M., Koenders, C., Altwegg, K., Combi, M. R., Glassmeier, K.-H., Gombosi, T. I., Hansen, K. C., Motschmann, U., Richter, I., Tenishev, V. M., and Tóth, G.: Plasma environment of a weak comet - Predictions for Comet 67P/Churyumov-Gerasimenko from multifluid-MHD and Hybrid models, Icarus, 242, 38-49, doi:10.1016/j.icarus.2014.07.021, 2014.

Sauer, K., Dubinin, E., Baumgärtel, K., and Tarasov, V.: Lowfrequency electromagnetic waves and instabilities within the Martian bi-ion plasma, Earth Planets Space, 50, 269-268, doi:10.1186/BF03352113, 1998.

Schlickeiser, R. and Yoon, P. H.: Electromagnetic fluctuations in magnetized plasmas. I. The rigorous relativistic kinetic theory, Phys. Plasmas, 22, 072108, doi:10.1063/1.4926828, 2015.

Schlickeiser, R., Ganz, A., Kolberg, U., and Yoon, P. H.: Electromagnetic fluctuations in magnetized plasmas II: Extension of the theory for parallel wave vectors, Phys. Plasmas, 22, 102111, doi:10.1063/1.4933210, 2015.

Silva, L. O., Bingham, R., Dawson, J. M., Mori, W. B., Mendonça, J. T., and Shukla, P. K.: Electroweak plasma instabilities and supernovae, Plasma Phys. Contr. F., 42, B223-B230, 2000.

Strickland, M.: The Chromo-Weibel Instability, Braz. J. Phys., 37, 76-766, doi:10.1590/S0103-97332007000500021, 2007.

Sturrock, P. A.: Kinematics of Growing Waves, Phys. Rev., 112, 1488-1503, doi:10.1103/PhysRev.112.1488, 1958.

Tátrallyay, M., Erdős, G., Balogh, A., and Dandouras, I.: The evolution of mirror type magnetic fluctuations in the magnetosheath based on multipoint observations, Adv. Space Res., 41, 15371544, doi:10.1016/j.asr.2007.03.039, 2008.

Treumann, R. A. and Baumjohann, W.: A note on the Weibel instability and thermal fluctuations, Ann. Geophys., 30, 427-431, doi:10.5194/angeo-30-427-2012, 2012.
Tsurutani, B. T. and Smith, E. J.: Strong hydromagnetic turbulence associated with Comet Giacobini-Zinner, Geophys. Res. Lett., 13, 259-262, doi:10.1029/GL013i003p00259, 1986.

Volwerk, M., Glassmeier, K.-H., Delva, M., Schmid, D., Koenders, C., Richter, I., and Szegö, K.: A comparison between VEGA 1, 2 and Giotto flybys of comet 1P/Halley: implications for Rosetta, Ann. Geophys., 32, 1441-1453, doi:10.5194/angeo-321441-2014, 2014.

Volwerk, M., Richter, I., Tsurutani, B., Götz, C., Altwegg, K., Broiles, T., Burch, J., Carr, C., Cupido, E., Delva, M., Dósa, M., Edberg, N. J. T., Eriksson, A., Henri, P., Koenders, C., Lebreton, J.-P., Mandt, K. E., Nilsson, H., Opitz, A., Rubin, M., Schwingenschuh, K., Stenberg Wieser, G., Szegö, K., Vallat, C., Vallieres, X., and Glassmeier, K.-H.: Mass-loading, pile-up, and mirror-mode waves at comet 67P/Churyumov-Gerasimenko, Ann. Geophys., 34, 1-15, doi:10.5194/angeo-34-1-2016, 2016.

Weibel, E. S.: Spontaneously Growing Transverse Waves in a Plasma Due to an Anisotropic Velocity Distribution, Phys. Rev. Lett., 2, 83-84, doi:10.1103/PhysRevLett.2.83, 1959.

$\mathrm{Wu}, \mathrm{C}$. S. and Davidson, R. C.: Electromagnetic instabilities produced by neutral-particle ionization in interplanetary space, J. Geophys. Res., 77, 5399, doi:10.1029/JA077i028p05399, 1972.

Wu, C. S., Winske, D., Papadopoulos, K., Zhou, Y. M., Tsai, S. T., and Guo, S. C.: A kinetic cross-field streaming instability, Phys. Fluids, 26, 1259-1267, doi:10.1063/1.864285, 1983.

Yoon, P. H. and Davidson, R. C.: Exact analytical model of the classical Weibel instability in a relativistic anisotropic plasma, Phys. Rev. A, 35, 2718-2721, doi:10.1103/PhysRevA.35.2718, 1987.

Yumoto, K., Saito, T., and Nakagawa, T.: Hydromagnetic waves near $\mathrm{O}(+)(\mathrm{OR} \mathrm{H} 2 \mathrm{O} /+/)$ ion cyclotron frequency observed by Sakigake at the closest approach to Comet Halley, Geophys. Res. Lett., 13, 825-828, doi:10.1029/GL013i008p00825, 1986. 